\title{
BH3-only proteins Puma and Beclin1 regulate autophagic death in neurons in response to Amyloid- $\beta$
}

\author{
Akash Saha ${ }^{1}$, Suraiya Saleem ${ }^{1,2}$, Ramesh Kumar Paidi ${ }^{1,3}$ and Subhas C. Biswas $\mathbb{D}^{1 凶}$ \\ (c) The Author(s) 2021, corrected publication 2021
}

\begin{abstract}
Alzheimer's disease (AD) is characterized by accumulation of senile amyloid- $\beta(A \beta)$ plaques and hyperphosphorylated tau tangles causing progressive loss of synapse and neuronal death. Out of the various neuron death modalities, autophagy and apoptosis are reported to be the major death paradigms in AD. However, how these two processes lead to neuronal loss is still inconspicuous. Here we report that under $A \beta$ toxicity, aberrant autophagy is induced with inefficient autophagic flux in neurons. Simultaneous activation of both autophagy and apoptosis are seen in primary cortical neurons as well as in transgenic mice brains. We found that induction of autophagy by rapamycin is detrimental for neurons; whereas downregulation of Beclin 1 , an important autophagy inducing protein, provides significant protection in $A \beta$ treated neuronal cells by blocking cytochrome-c release from the mitochondria. We further report that downregulation of Puma, a BH3-only pro-apoptotic protein, inhibits the induction of aberrant autophagy and also ameliorates the autophagy flux under the influence of $A \beta$. Notably, stereotactic administration of shRNAs against Puma and Beclin 1 in adult $A \beta$-infused rat brains inhibits both apoptotic and autophagic pathways. The regulation of both of the death processes is brought about by the direct interaction between Puma and Beclin 1 upon $A \beta$ treatment. We conclude that both Beclin 1 and Puma play essential roles in the neuronal death caused by the induction of aberrant autophagy in AD and targeting their interaction could be vital to understand the crosstalk of autophagy and apoptosis as well as to develop a potential therapeutic strategy in AD.
\end{abstract}

Cell Death Discovery (2021)7:356; https://doi.org/10.1038/s41420-021-00748-x

\section{INTRODUCTION}

The underlying pathogenesis of Alzheimer's disease (AD) is selective synaptic loss followed by the death of neurons leading to memory impairment and dementia. The most accepted hypothesis on disease pathogenesis is the amyloid cascade hypothesis which suggests that the earliest pathological hallmark of the disease is the deposition of amyloid- $\beta(A \beta)$ plaques that subsequently leads to tau deposition, neuronal loss, synaptic anomalies, and degeneration [1, 2]. This hypothesis is extended by Bart de Strooper and Eric Karran [3] and they proposed that the accumulation of plaques and tau tangle is a slow and gradual process that acts as a risk factor. The clinical manifestation of $A D$ only occurs when there is a failure in the cellular homeostasis and is denoted by dysfunctional neurovascular unit, anomalous neuronal circuitry, aberrant microglial and astrocyte functions which altogether lead to neurodegeneration [4]. The two vital cell death mechanisms that influence neurodegeneration are apoptosis and autophagy [5-7].

Autophagy is a major intracellular catabolic pathway that eliminates damaged cellular contents like proteins, lipids, and other organelles, with the help of lysosomal enzymes [8-10]. Neurons are highly dependent on autophagy for their survival due to their extreme polarized and post-mitotic nature [11]. Autophagy maintains the health of neurons by preventing the accumulation of aggregated cytosolic wastes of proteins or membranes [12]. Accumulation of cellular wastes in neurons may incur a great burden in the cells which are not diluted due to their non-dividing nature $[13,14]$. Knockout of Atg genes such as ATG5 [15] or ATG7 [16] are shown to be embryonically lethal and displayed behavioral anomaly in mice [17]. Genome-wide sequencing showed that a few genes like ATG5, ATG7, ATG101, and ATG16L1 are indispensable for neuronal survival [18]. However, the role of autophagy in $A D$ is quite controversial. Accumulation of autophagy vacuoles (AVs) is reported in $A D$ patients' brains as compared to normal brain biopsies [19]. While few reports suggest autophagy being crucial in the degradation of A $\beta$ plaques, APP [20-24] and tau tangles [25-27], there are many reports suggesting the presence of aberrant $A V s$ containing $A \beta$ aggregates in AD brains [28-31]. Since there are varied reports suggesting the beneficial and deteriorating effects of autophagy in $A D$, its exact role in $A D$ pathogenesis still remains uncertain.

In $A D$, it is reported that the $A \beta$ plaque may generate oxidative stress or trigger expression/activation of a number of proapoptotic proteins in neurons leading to cell death by apoptosis [32-36]. Reports of increased DNA fragmentation, granulated chromatin, changes in cell shape and size, caspase activity, changes in levels of the apoptosis-related proteins of the $\mathrm{BCl}-2$ family have been cited in the AD patients' brains bearing senile

\footnotetext{
${ }^{1}$ Cell Biology and Physiology Division, CSIR-Indian Institute of Chemical Biology, 4 Raja S. C. Mullick Road, Kolkata 700 032, India. ${ }^{2}$ Present address: Suraiya Saleem, Bhupat and Jyoti Mehta School of Biosciences, Indian Institute of Technology Madras, IIT P.O., Chennai 600 036, India. ${ }^{3}$ Present address: Department of Neurological Sciences, RUSH University Medical Center, Chicago, IL 60612, USA. ${ }^{凶}$ email: biswassc@gmail.com
}

Received: 1 September 2021 Revised: 22 October 2021 Accepted: 25 October 2021

Published online: 15 November 2021 
plaque depositions $[37,38]$. Bcl-2 protein family play important roles in the interplay of autophagy and apoptosis [39, 40]. Reports suggest that $\mathrm{BH} 3$-domain only pro-apoptotic protein, Bim, sequesters Beclin1 or ATG6, which is a vital autophagic protein and inhibits autophagic initiation in starved HeLa cells [41]. Puma (p53 upregulated modulator of apoptosis) is a potent proapoptotic protein like Bim; however, its role in autophagy is not yet revealed. $\mathrm{BCl}-2$ is supposed to have a dual characteristic; it is an anti-apoptotic protein but can also act as an anti-autophagic protein when it inhibits Beclin1 from associating with the autophagic machinery $[39,42]$. Another level of crosstalk is served by the caspases. Wirawan and group identified two caspase-3 cleavage sites on Beclin1 [43] and the cleaved part is shown to sensitize the mitochondria for apoptosis. The C-terminal fragment of Beclin1 is reported to translocate to the mitochondria thus releasing apoptotic signals [44]. There are many levels of regulations and interactions between the autophagic and apoptotic machineries which are not yet clear. In this study, we concentrate primarily on the activation of both autophagy and apoptosis in neurons under the influence of $A \beta$ and find out the role of autophagy in determining the neuronal fate. We specifically look into the effect of $\mathrm{BH} 3$-only proteins, Puma and Beclin1 in regulating autophagic death of neurons under $A \beta$ toxicity.

\section{RESULTS}

\section{$A \beta$ induces both autophagy and apoptosis}

We began our quest using neuronally differentiated PC12 cells being treated with oligomeric $A \beta$ at a concentration of $5 \mu \mathrm{M}$ for overnight (Supplementary Data, Fig. S1A-B). We checked the onset and progression of autophagy and apoptosis by checking the levels of their markers by immunocytochemistry. We found an increase in the expression of autophagosome markers such as LC3, p62, a lysosomal marker, Lamp1, and a marker of DNA double-strand breaks, $\mathrm{pH} 2 \mathrm{AX}$ in differentiated $\mathrm{PC} 12$ cells under $A \beta$ toxicity over a period of $0-24 \mathrm{~h}$ (Fig. 1A-E). The expression of these proteins gradually increased over time. We also checked for apoptosis by TUNEL assay under the same condition and obtained similar results (Supplementary Data, Fig. S2A-B). This upregulation of autophagic and apoptotic proteins were further checked by western blotting and an increase of LC3B, p62, Lamp1, and $\mathrm{pH} 2 \mathrm{AX}$ levels was found as early as $8 \mathrm{~h}$ with a peak at $16 \mathrm{~h}$ (LC3B and $\mathrm{p} 62$ ) or $24 \mathrm{~h}$ (Lamp1 and $\mathrm{pH} 2 \mathrm{AX}$ ) post $\mathrm{A} \beta$ treatment (Fig. 1F-M). Having observed induction of both autophagy and apoptosis after $A \beta$ treatment, we were intrigued to investigate the specific cellular population in which both these phenomena occurred simultaneously. Our coimmunocytochemistry studies revealed that following the $A \beta$ insult, there was a gradual increase of cells exhibiting both LC3 and $\mathrm{pH} 2 \mathrm{AX}$, and the number of cells showing co-staining increased significantly in a time-dependent manner from $8 \mathrm{~h}$ to $24 \mathrm{~h}$ of treatment (Fig. $1 \mathrm{~N}, \mathrm{O}$ ).

Since PC12 cells is an immortal cell line, we checked the status of autophagic and apoptotic proteins in primary cortical neurons. We found a significant and gradual increase in the expressions of autophagic initiation marker, LC3B under $A \beta$ insult in a time-dependent manner as checked by both immunocytochemistry and western blotting (Fig. 2A-D). Protein levels of another important autophagic protein, Beclin1, and autophagy flux marker, p62 were also elevated gradually and significantly under $A \beta$ toxicity in cortical neurons from $8 \mathrm{~h}$ to $24 \mathrm{~h}$ (Fig. 2E-H). The apoptotic effector, cleaved caspase-3 staining was also shown to be increased under the influence of $\mathrm{A} \beta$ from $8 \mathrm{~h}$ to $24 \mathrm{~h}$ (Fig. $2 \mathrm{l}, \mathrm{J}$ ). Collectively, these data indicate that both apoptosis and autophagy are induced upon $A \beta$ insult in neuronal cells and both phenomena occur simultaneously in a particular cell.
Induction of autophagy does not protect cells from $A \boldsymbol{\beta}$ toxicity Since we found that both autophagy and apoptosis are induced in response to $A \beta$ treatment in neuronal cells, we therefore intended to check if autophagy is beneficial on cell viability in presence of $A \beta$. We treated neuronally differentiated PC12 cells with $A \beta$ for overnight in the presence of pan-caspase inhibitor (zVAD-FMK) to inhibit apoptosis, rapamycin to induce autophagy, or both zVADFMK (to inhibit apoptosis) and rapamycin (to simultaneously induce autophagy). Results showed that the death caused by $A \beta$ was rescued when apoptosis was inhibited by zVAD-FMK. However, there was no rescue seen when autophagy was induced using rapamycin. Further, simultaneous induction of autophagy and inhibition of apoptosis by rapamycin and zVAD-FMK respectively did not provide any more significant protection as compared to when only apoptosis was blocked, suggesting that induction of autophagy does not play a major role in cell survival under $A \beta$ insult, at least in cultured neurons (Fig. 3A-B).

\section{Beclin 1 promotes neuronal cell death upon $A \boldsymbol{\beta}$ treatment}

Beclin1 is a major autophagy protein that has been reported to be induced under neurodegenerative conditions $[53,54]$ and plays an essential role in autophagy-apoptosis crosstalk [55]. Interestingly, recent evidences have also shown that proteolytic cleavage of Beclin1 causes apoptotic neuronal loss in two different models of neurodegeneration [56]. Since our previous experiments showed that autophagy induction does not protect neurons from $A \beta$ toxicity, we next checked whether inhibition of autophagy by downregulating Beclin1 could provide protection to neurons. Neuronally differentiated $\mathrm{PC} 12$ cells were transfected with either shBeclin1 or shRand (control), and then were subjected to $A \beta$ treatment (Fig. 4A, B) for $24 \mathrm{~h}$ and $48 \mathrm{~h}$. Results showed significant protection, where Beclin1 was downregulated compared to cells transfected with shRand. Similar protection was obtained in cortical neurons treated with $1.5 \mu \mathrm{M} \mathrm{A \beta}$ upto $48 \mathrm{~h}$ (Fig. 4C, D). Knockdown of Beclin 1 not only protects neuronal cells from death but also retains the overall neuronal morphology of neurons even after $48 \mathrm{~h}$ of $A \beta$ treatment (Fig. 4C). We further observed that number of cells in which Beclin1 was downregulated, levels of cytochrome-c were significantly low as opposed to control plasmid transfected cells upon treatment with $A \beta$ (Fig. 4E, F). Taken together, these results indicate that Beclin1 plays an essential role in neuron death evoked by $A \beta$ by inducing both autophagy and apoptosis.

\section{Puma and FoxO3a evoke autophagy initiation but hinder autophagy flux in neuronal cells upon $A \beta$ insult}

We have previously shown the activation of Puma in response to $A \beta$ in neurons leads to neuronal death [34] and it has been also shown that Puma plays an essential role in the regulation of mitophagy in cancerous cells [57]. Taking a cue from these studies, we checked the effect of downregulation of Puma, on the regulation of autophagy initiation and flux under $A \beta$ toxicity. We observed a significant drop in the levels of LC3B (Fig. 5A, B) and p62 (Fig. 5C, D) in the differentiated PC12 cells in which Puma had been downregulated as compared to the control cells, suggesting that downregulation of Puma inhibited the autophagy initiation but helped in maintaining the autophagic flux, as indicated by the reduced levels of $p 62$, which is otherwise seen to be accumulated and upregulated under the influence of $A \beta$. Since Puma is directly activated by a transcription factor FoxO3a [33, 34] and FoxO3a is known to play a major role in autophagy induction in cardiomyocytes for normal cardiac functioning [58], we checked if this transcription factor plays any role in autophagy initiation and maintaining the autophagy. Performing an immunocytochemistry analysis in FoxO3a downregulated PC12 cells, we found a significant decrease in autophagic initiation (Fig. 5E, F) and p62 accumulation (Fig. 5G, H) as compared to the control cells under $A \beta$ toxicity. We also determined these protein levels by western 


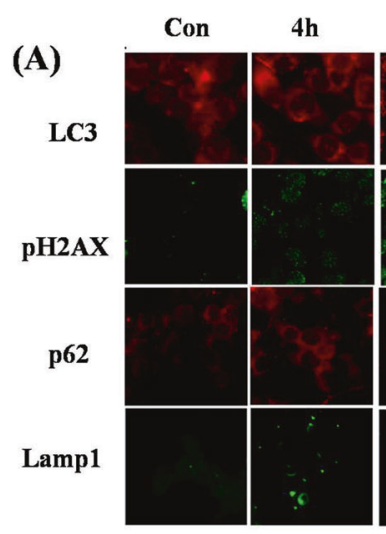

(D)

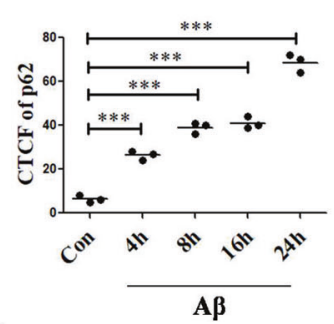

(N)

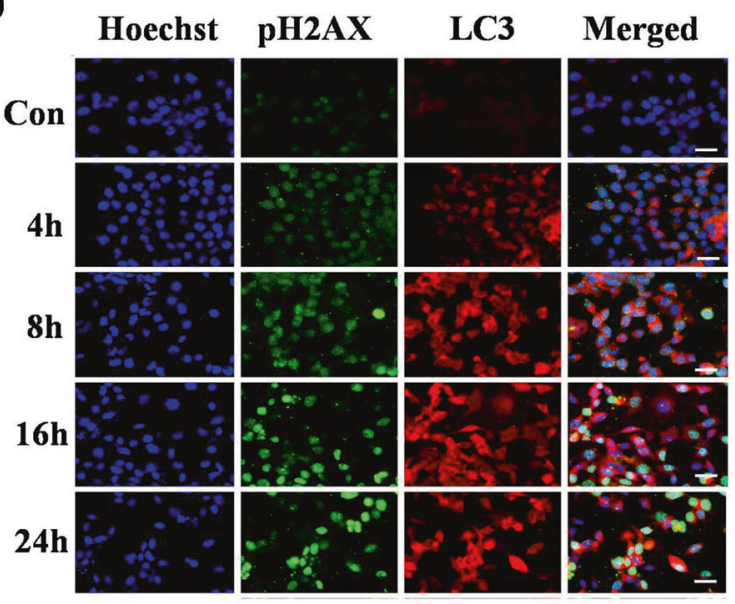

(E)

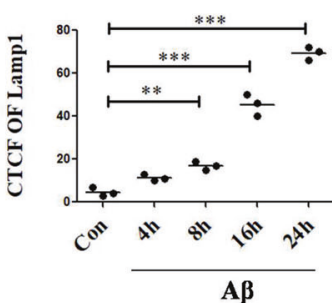

(B)
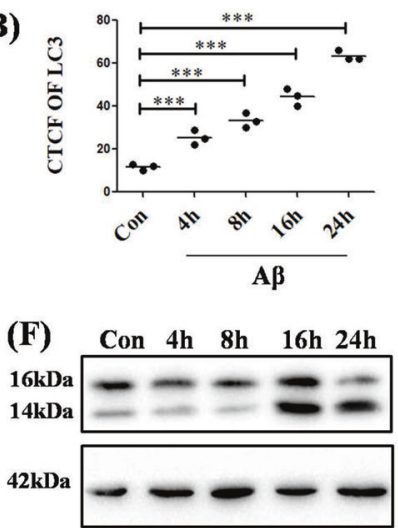

(H)

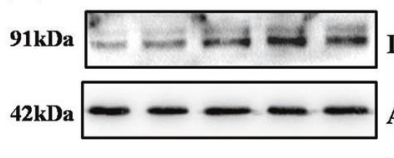

(J)
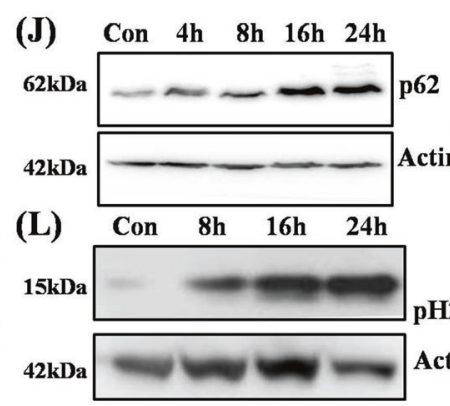

(O)

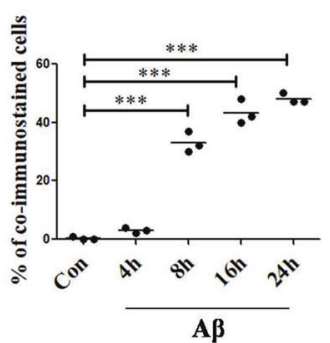

(C)

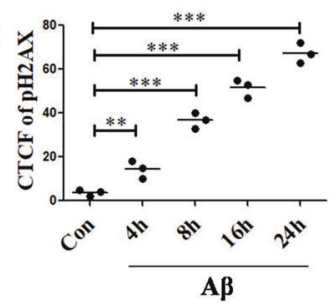

(G)

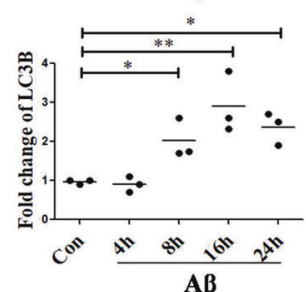

(I)

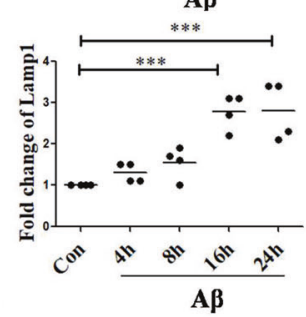

(K)

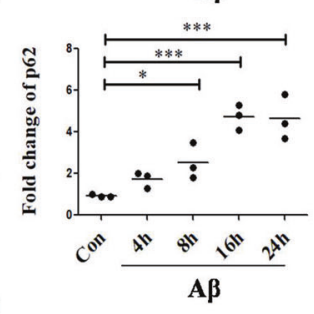

(M)

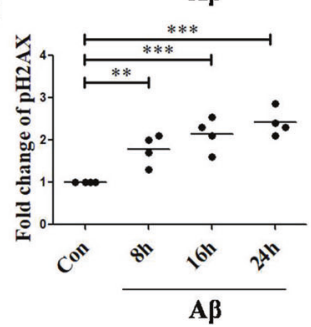

Fig. 1 A $\beta$ treatment induces both autophagy nad apoptosis in differentiated PC12 cells. (A) PC12 cells were primed and treated with $5 \mu M$ $\mathrm{A} \beta$ and stained with autophagy markers: LC3 (red), p62 (red), and Lamp1 (green) and apoptotic marker: pH2AX (green) in separate sets of experiments. Scale bars: $50 \mu \mathrm{m}$. (B), (C), (D), and (E) represents the corrected total cell fluorescence of LC3, pH2AX, p62, and Lamp1 respectively. Data represented were collected from 3 independent experiments. PC 12 cells were primed and treated with $5 \mu \mathrm{M} A \beta$. Cell lystaes were used to asses the endogenous levels of $(\mathbf{F})$ LC3B, (H) Lamp1, $(\mathbf{J})$ p62, and $(\mathbf{L})$ pH2AX respectively for the time points as indicated. (G), $(\mathbf{I})$, $(\mathbf{K})$, and $(\mathbf{M})$ are the graphical representation of the fold change of the protein levels of LC3B, Lamp1, p62, and pH2AX respectively under A $\beta$ toxicity. Data represented were collected from 3 independent experiments, except for (I) and (M) where data represented was from 4 independent experiments. (N) Primed PC12 cells were treated with $5 \mu \mathrm{M}$ of $\mathrm{A} \beta$ and were stained with anti-LC3B antibody (red) and anti-pH2AX antibody (green) for co-immunocytochemistry studies. Nuclei were stained with Hoechst (blue). Scale bar $50 \mu \mathrm{m}$. (0) Graphical representation of the percentage co-immunostained cells under A $\beta$ toxicity. Data represented were collected from 3 independent experiments. The asterisks denote statistically significant differences from control at corresponding time points: $p<0.05,{ }^{* *} p<0.001,{ }^{* * *} p<0.0001$.

blotting and found a decrease in the levels of LC3B (Fig. 5I-L) and p62 (Fig. 5M-P) in both shPuma and shFoxO3a transfected PC12 cells as compared to control transfected cells under $A \beta$ treatment, indicating a dual role of both Puma and FoxO3a in the regulation of autophagy and apoptosis.

\section{Autophagy and Apoptosis is induced simultaneously in brains of $5 x F A D$ mice}

To validate our in vitro studies on the occurrence of autophagy and apoptosis under $A \beta$ toxicity, we first checked the autophagy levels in vivo in 12 months APP/PS1 transgenic mice brain and found an increase in the levels of both LC3 and p62 as compared to the wild type control mice brain (Supplementary Data, Fig. S3A-D). Next, we checked for the advent of autophagy and apoptosis in 6 months old 5xFAD mice brain as compared to the age-matched wild-type mice brain. Our immunohistochemistry studies show that there is an increase in the expression of autophagy marker proteins LC3 (Fig. 6B, C) and Lamp1 (Fig. 6D, E), in the 5xFAD mice brain compared to wild type. Also, we found an increase in the staining of Puma in the 5xFAD brain as compared to wild-type brains (Fig. 6F, G). Further, protein levels of autophagy markers were checked by western blot and we found significantly elevated levels of both LC3B and Lamp1 in 6 months old 5xFAD mice brain tissue lysates from the cortex (Fig. $6 \mathrm{H}-\mathrm{K}$ ) and 

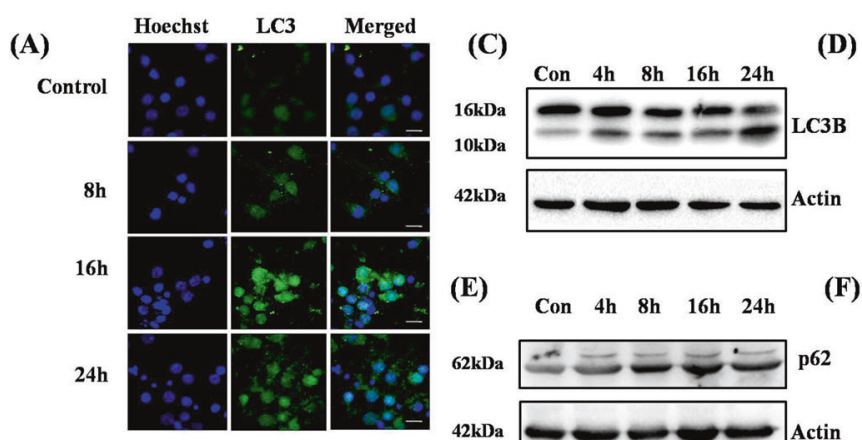

(E)

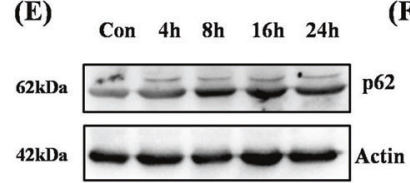

(B)

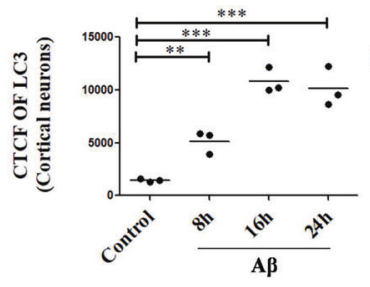

(G)

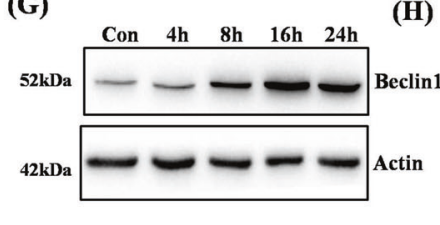

H)
(D)

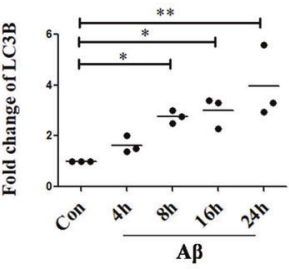

(F)
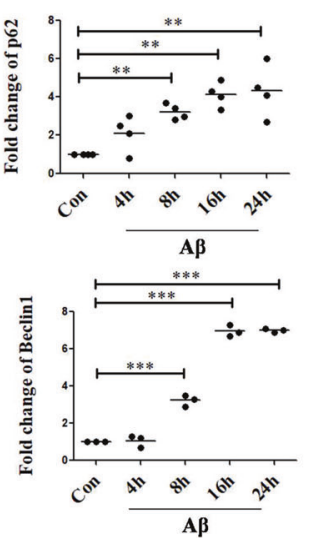

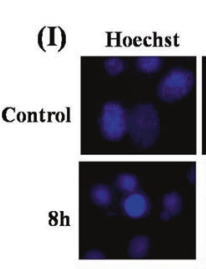

Cleaved

caspase 3

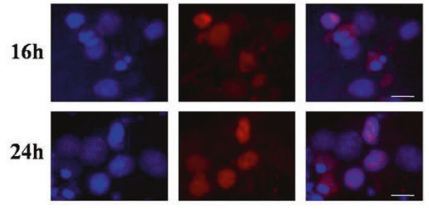

(J)

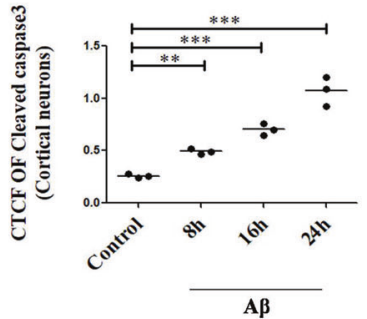

Fig. $2 A \beta$ evokes autophagy and apoptosis in primary cortical neurons. (A) Primary rat cortical neurons (7DIV) were treated with 1.5 $\mu \mathrm{M} A \beta$ for indicated time periods and stained with an anti-LC3B antibody (green) to assess the endogenous levels of the protein. Nuclei were stained using Hoechst (blue). Scale bars: $50 \mu \mathrm{m}$. (B) Graphical representation of the corrected total cell fluorescence of LC3B. Data represented were collected from 3 independent experiments. Primary rat cortical neurons (7DIV) were treated with $1.5 \mu \mathrm{M}$ A $\beta$ for indicated time periods and the cell lysates were subjected to western blot analysis to check the levels of $(\mathbf{C})$ LC3B, (E) p62, and (G) Beclin1 respectively. (D), (F) and (H) are the graphical representations of the fold change of the protein levels of LC3B, p62, and Beclin 1 respectively. Data represented were collected from 3 independent experiments, except for (F) where data represented was from 4 independent experiments. (I) Primary rat cortical neurons (7DIV) were treated with $1.5 \mu \mathrm{M} \mathrm{A} \beta$ for indicated time periods and stained with an anti-cleaved caspase-3 antibody (red) to assess the endogenous levels of cleaved caspase-3. Nuclei were stained using Hoechst (blue). Scale bars: $50 \mu \mathrm{m}$. (J) Graphical representation of the corrected total cell fluorescence of cleaved caspase-3. Data represented were collected from 3 independent experiments. The asterisks denote statistically significant differences from control at corresponding time points; ${ }^{*} p<0.05,{ }^{* *} p<0.001,{ }^{* * *} p<0.0001$.

hippocampus (Fig. 6L, O) as compared to the age-matched wild type mice cortex and hippocampus lysates respectively. In order to check if both the processes, viz, autophagy and apoptosis occurred simultaneously in the same cells, we performed the immunohistochemistry and TUNEL assays together in the same brain sections of 5xFAD mice and in corresponding wild type sections. We found an increase in the levels of LC3 and TUNEL positive cells in 5xFAD mice as compared to the wild-type set. Interestingly we also observed that many cells underwent both autophagy and apoptosis simultaneously in 5xFAD (Fig. 6P, Q). We thereby confirm that both autophagy and apoptosis simultaneously occur in the brain of $A D$ mice. The level of crosstalk between both these processes may finally determine the survival or death of neurons.

\section{Down regulation of Puma and Beclin1 leads to inhibition of both autophagy and apoptosis in vivo}

Since both autophagy and apoptosis are triggered simultaneously in both cellular and animal models of $A D$, we next checked the role of two key BH3-only proteins, Beclin1 (essential for autophagosome formation) and Puma (a pro-apoptotic protein) in cell death in an in vivo model. We created an animal model of $A D$ by injecting oligomeric $A \beta$ in the cortex of rat brains by stereotaxy. Puma and Beclin1 were downregulated in $A \beta$-infused rats by injecting specific shRNAs against them, exclusively. We infused reverse $A \beta$ peptide $(\mathrm{rA} \beta)$ in rat brains, to create negative controls and we infused PBS in the rat brains for control, in similar locations. We took the tissue lysates of the cortex and hippocampus of the brains of these rats and performed western blotting where we found a significant reduction in the levels of autophagy proteins LC3B and p62 in the cortex (Fig. 7A-D) and hippocampus(Fig. 7E-H), in both Puma or Beclin1 knockdown rat brains as compared to the $A \beta$-infused rat brains. We also found a decrease in the cleaved PARP levels in the brains of rats where we downregulated Puma or Beclin1 as compared to that of the $A \beta$-infused rat brains (Fig. 7A, B, E, F), suggesting that both Puma and Beclin1 play an essential role in the regulation of both autophagy and apoptosis, in determining the fate of neurons in AD.

\section{Autophagic BH3-only protein Beclin1 interacts with pro- apoptotic BH3-only protein Puma}

Structural studies indicate that Beclin1 is a $\mathrm{BH} 3$-only protein with a $\mathrm{BH} 3$ binding motif through which it interacts with $\mathrm{BCl}-2 / \mathrm{BCl}-\mathrm{xL}$ [59]. Reports have prompted towards a close interaction between $\mathrm{BH} 3-$ only proteins Bim and Beclin1 which holds the essence of regulation of autophagy in starved conditions [41]. Taking a cue from such reports, we investigated whether Puma, due to the presence of $\mathrm{BH} 3$ motif, interacted with Beclin1 at any level in our model. Since we found that downregulation of Puma or Beclin1 leads to a substantial amount of inhibition of autophagy as well as apoptosis, it was imperative to have checked whether these proteins show any kind of interaction between them or not. We performed immunoprecipitation studies on tissue lysates of cortical neurons. Our study suggested a potential interaction between Puma and Beclin1. Furthermore, we see an increase in the interaction of the two $\mathrm{BH} 3$-only proteins under $A \beta$ treated conditions as compared to the control (Fig. 8A-D). Collectively, these findings hint towards the fact that pro-apoptotic protein Puma regulates autophagy by its close association with Beclin1 which is an important autophagy protein (Fig. 8E). Downregulation of any of these proteins blocks initiation of autophagy and accumulation of cargo leading to the survival of neurons.

\section{DISCUSSION}

In this study, we investigated whether two $\mathrm{BH}$-only proteins Beclin1, an inducer of autophagy, and Puma, a pro-apoptotic 


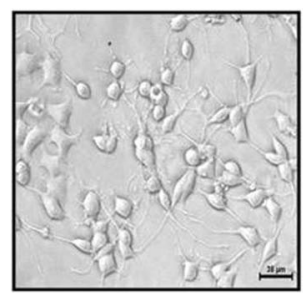

Control

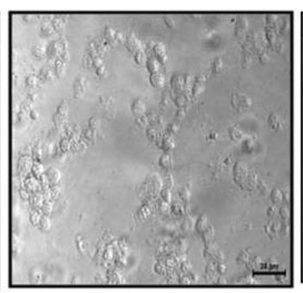

$\mathbf{A} \boldsymbol{\beta}$

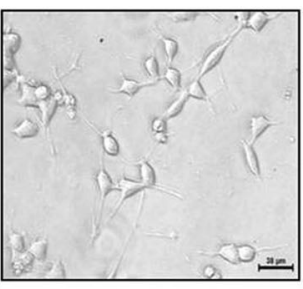

A $\beta$ + zVAD-FMK

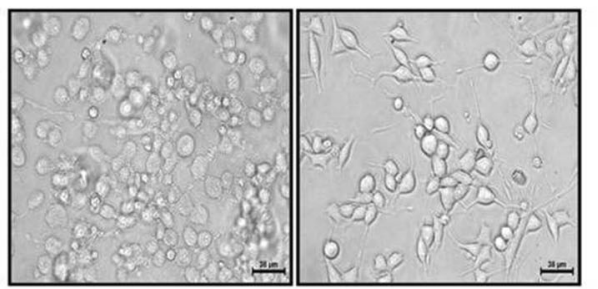

$\mathbf{A} \boldsymbol{\beta}+$

Rapamycin
$\mathbf{A} \boldsymbol{\beta}+$ Rapamycin + zVAD-FMK

(A)

(B)
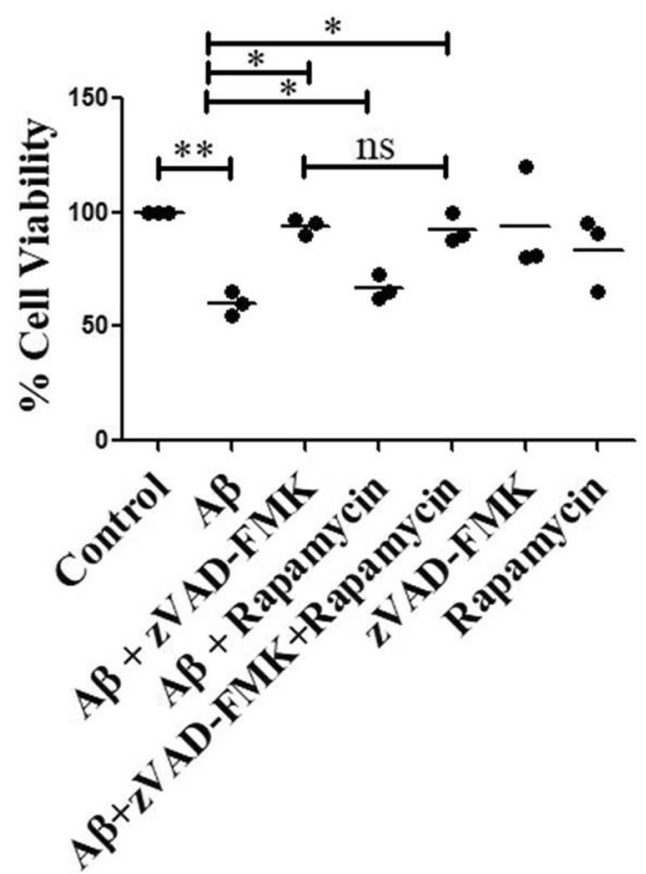

Fig. 3 Activation of autophagy does not provide protection against $\mathbf{A} \boldsymbol{\beta}$ treatment. (A) $\mathrm{PC} 12$ cells were primed and treated with $5 \mu \mathrm{M}$ A $\beta$ in the presence of autophagy inducer rapamycin; pan-caspase inhibitor zVAD-FMK and in conjunction of rapamycin and zVAD-FMK for $16 \mathrm{~h}$ and phase-contrast micrographs were taken. Scale bars: $38 \mu \mathrm{m}$. (B) Graphical representation of cell survival following A $\beta$ treatment with zVAD-FMK, rapamycin, or both, for $16 \mathrm{~h}$. Data represented were collected from 3 independent experiments. The asterisks denote statistically significant differences from control at corresponding conditions; ${ }^{*} p<0.05,{ }^{* *} p<0.001$, ns represents no significance.

protein, interact with each other and influence neuron death in AD.A set of experimental findings suggest that both Beclin 1 and Puma binds with each other and play necessary roles in autophagy induction but are responsible for impaired autophagy flux and apoptotic death of neurons. First, both autophagy and apoptosis are induced simultaneously in neurons upon $A \beta$ exposure and in transgenic AD mice. Autophagolysosomes formation is induced as determined by the increase in levels of LC3B and Lamp1, however autophagy flux is reduced as p62 levels are increased. The induction of apoptosis is determined by the levels of $\mathrm{pH} 2 \mathrm{AX}$. Second, induction of autophagy is not beneficial for neurons as rapamycin does not block neuron death evoked by $A \beta$. However, inhibition of autophagy by downregulating Beclin1 protects neurons from death induced by $A \beta$. Third, Puma and its transcriptional regulator FoxO3a, play an essential role in autophagy induction and impaired autophagy flux. Fourth, both Beclin 1 and Puma are responsible for the induction of autophagy and reduced autophagy flux in the $A \beta$-infused rat brain. Importantly, both are essential for PARP cleavage, an important marker of caspase-dependent apoptosis. Finally, Beclin1 and Puma directly interact with each other and this interaction is increased in response to $A \beta$ treatment.
Besides Beclin1, Atg5 is reported to play a major role in the autophagy-apoptosis crosstalk. It is reported that Atg5 can trigger autophagic cell death by interacting with FADD which promotes apoptosis [60]. Evidences show that Atg5 is cleaved by calpains and the $\mathrm{N}$-terminal fragment reaches the mitochondria and induces apoptosis by releasing cytochrome-c and activation of caspases [61]. Puma has shown to be regulated by autophagy in Tumor Necrosis factor-related Apoptosis-Inducing Ligand (TRAIL) mediated cancer cell death [62]. Alternatively, Puma plays a necessary role in protein clearance in neurons in response to pesticide, chlorpyrifos [63]. Puma has also been shown to play an essential role in mitophagy in cancer cells [57]. Moreover, both autophagic and apoptotic features are seen in dying neurons in cases of neonatal hypoxia-ischemia [64]. Along with the occurrence of the aforesaid processes, we now show that both autophagy and apoptosis occur simultaneously in neurons, and Beclin1 and Puma play an essential role on the induction of autophagy and apoptosis in $A \beta$ induced neurodegeneration.

Although we found an induction of autophagy in neuronal cells, the accumulation of autophagy residual aggregates, as marked by p62, takes place at very early stages upon $A \beta$ toxicity. Nixon's group noted the accumulation of subcellular aggregates and AVs in dystrophic neuritis [65] and further studies have supported the 


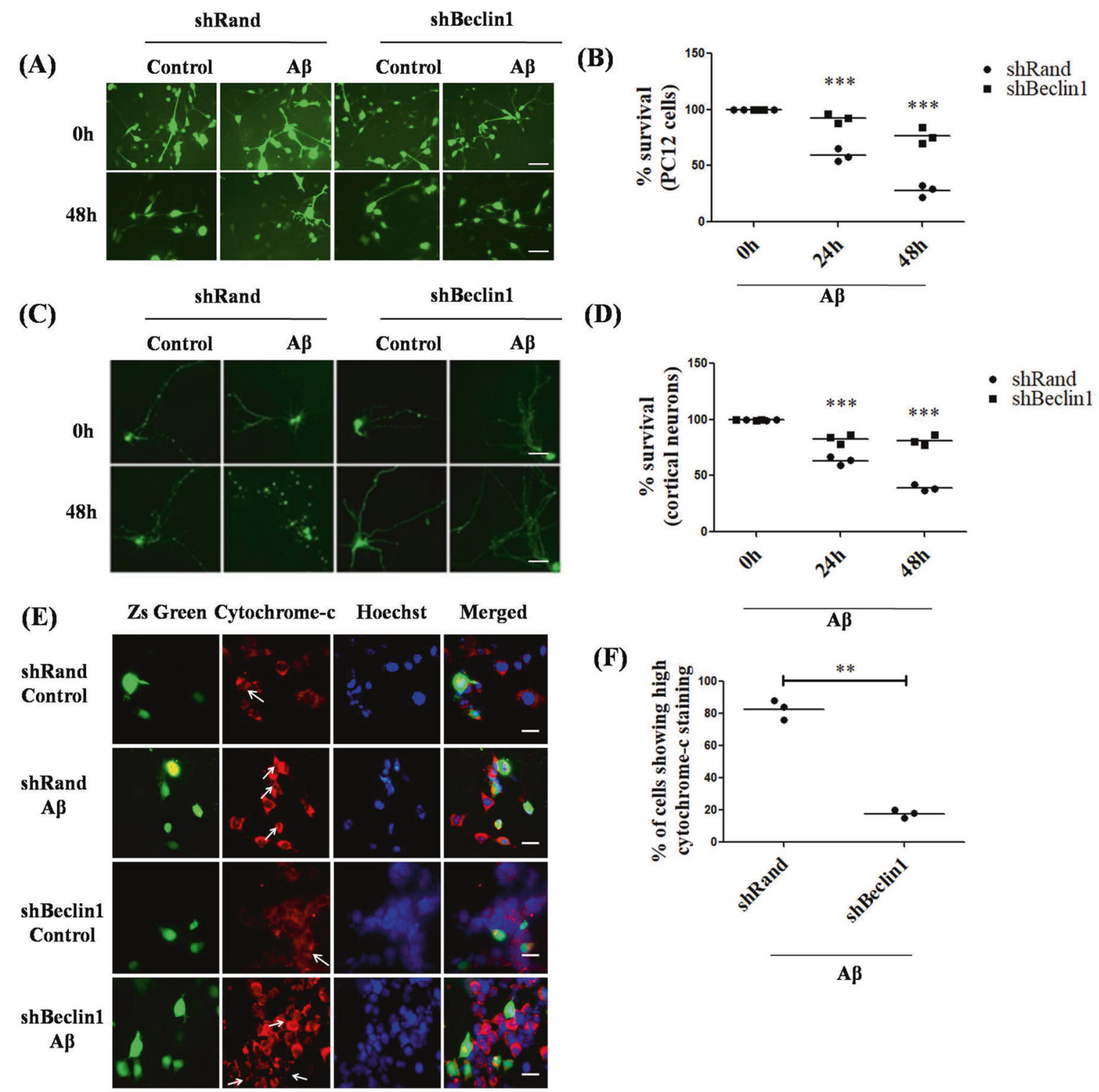

Fig. 4 Downregulating Beclin1 protects neuronal cells against A $\boldsymbol{\beta}$ treatment. (A) Neuronally differentiated PC12 cells were transfected with shBeclin1-ZsGreen or shRand-ZsGreen. $48 \mathrm{~h}$ post transfection, cells were treated with $5 \mu \mathrm{M} A \beta$. Representative images of transfected cells that were maintained in the presence or absence of $A \beta$ for indicated time periods are shown. Scale bars: $50 \mu \mathrm{m}$. (B) Graphical representation of the percentage of viable cells where live green cells were counted just after treatment and after $48 \mathrm{~h}$ of treatment with $A \beta$ under a fluorescence microscope. Data represented were collected from 3 independent experiments. (C) Primary cultured rat cortical neurons (3 DIV) were transfected with shBeclin1-ZsGreen or control shRand-ZsGreen and maintained for $48 \mathrm{~h}$ and then subjected to $1.5 \mu \mathrm{M} A \beta$ for $48 \mathrm{~h}$. Representative pictures of transfected neurons that were maintained in the presence or absence of $A \beta$ for indicated time periods are shown. Images were taken using an inverted fluorescence microscope. Scale bars: $50 \mu \mathrm{m}$. (D) Graphical representation of the percentage of viable green cells after each time point. The numbers of surviving transfected (green) cells were counted under a fluorescence microscope just after treatment and after $48 \mathrm{~h}$ of treatment with $\mathrm{A} \beta$. Data represented were collected from 3 independent experiments. (E) Neuronal PC12 cells were transfected with shBeclin1-ZsGreen or shRand-ZsGreen. $48 \mathrm{~h}$ post-transfection, cells were treated with $A \beta$ and immunostained for cytochrome-c and checked under a fluorescence microscope. Scale bars: $50 \mu \mathrm{m}$. (F) Graphical representation of the percentage of cells with high cytochrome-c staining after A $\beta$ treatment in Beclin1 downregulated conditions. Data represented were collected from 3 independent experiments. The asterisks denote statistically significant differences from control (shRand) at corresponding time points or conditions; ${ }^{* *} p<0.001,{ }^{* * *} p<0.0001$.

accumulation of these AVs associated with dying neuritis in APP/ PS1 mice [66]. In other studies, Nixon's group showed that apoptosis was the mode of neuronal death in aging APP/PS mice and was the source of crosstalk between apoptosis and autophagy [67]. Consistent with that, we also observed the increase in levels of autophagy in APP/PS1 mice brain. Additionally, we found simultaneous activation of both autophagy and apoptosis in 6 months old 5xFAD mice brain compared to the age-matched wild-type mice brains.
Further we observed that induction of autophagy by rapamycin, which inhibits mTOR - the master regulator of autophagy, provides no beneficial effect on the viability of cells under $A \beta$ treatment; on the contrary, inhibiting autophagy with 3-Methyladenine provided some protection to neurons [68]. As expected, inhibiting apoptosis by zVAD-FMK significantly helped in cell viability. However, there was no synergistic effect seen in cell survivability under apoptosis inhibited-autophagy induced conditions compared to apoptosis inhibited conditions. To further establish our findings on the negative 
(A)

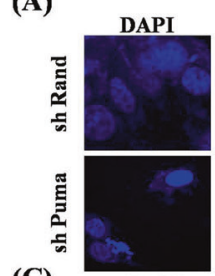

(C)

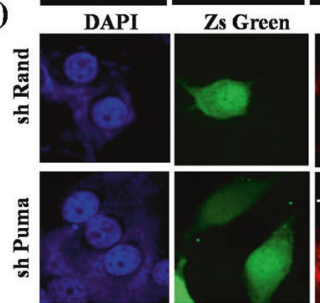

(E)

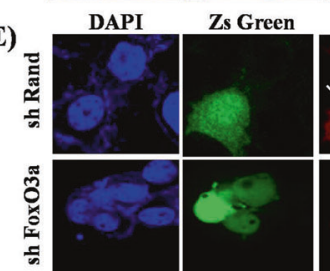

(G)

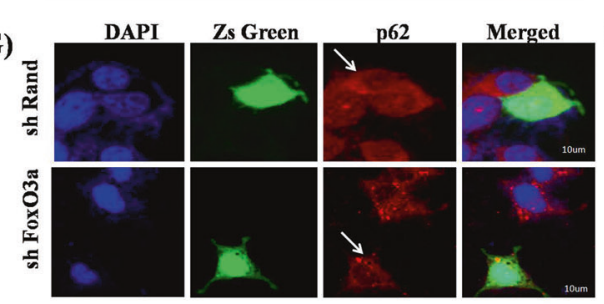

(B)
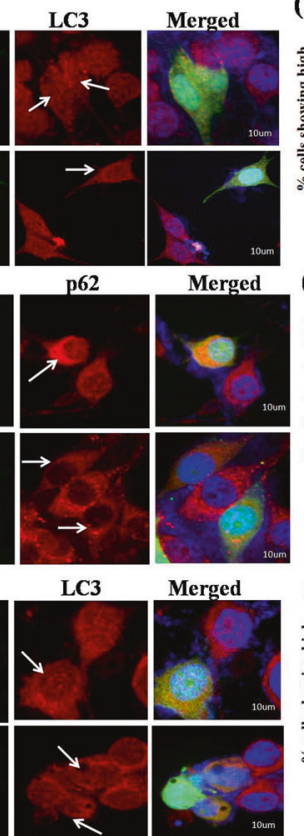
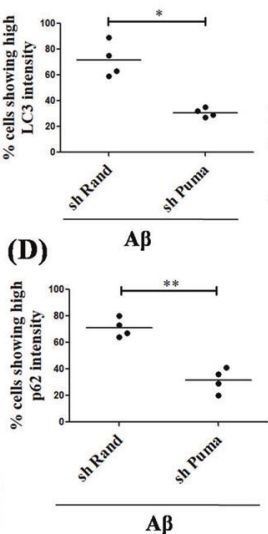

(F)

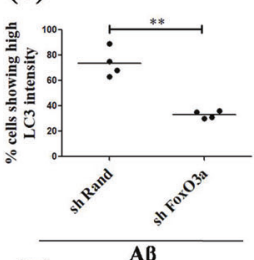

(H)

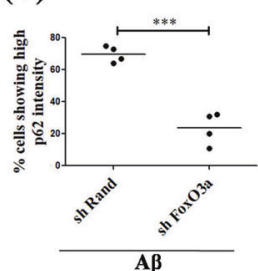

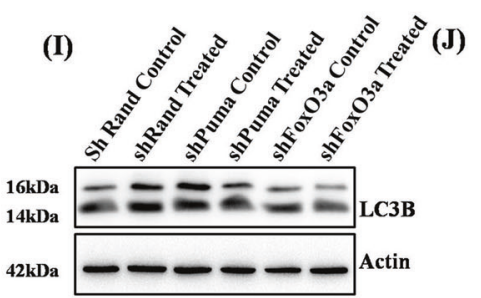

(K)

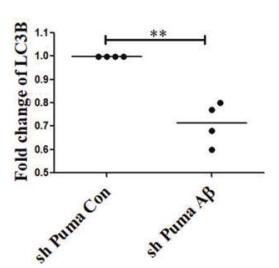

(L)
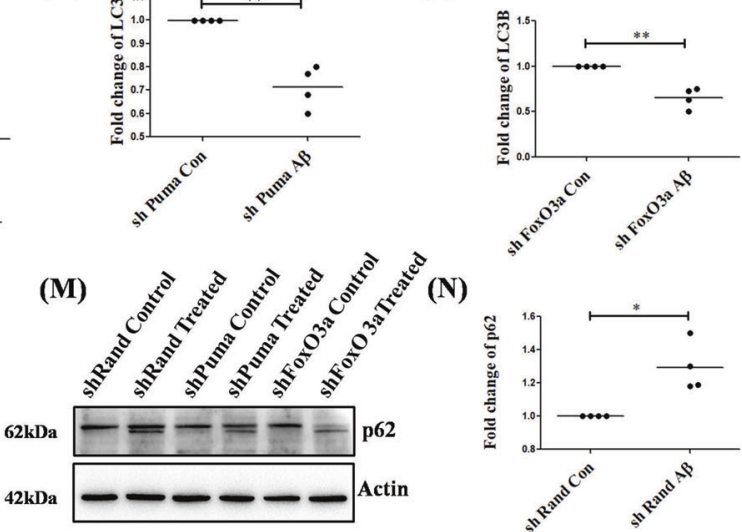

(N)

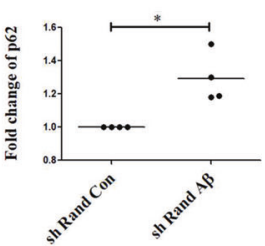

(O)

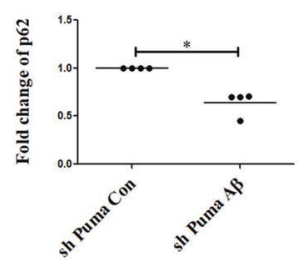

(P)

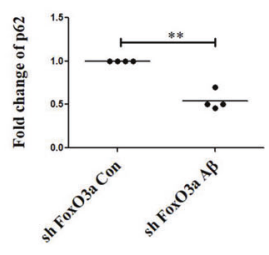

Fig. 5 Puma and FoxO3a trigger autophagy initiation but interferes in autophagy flux upon A $\beta$ toxicity. Naïve PC12 cells were transfected with shPuma-ZsGreen (green) or shRand-ZsGreen (green), primed, and then treated with $5 \mu \mathrm{M} A \beta$ for overnight after which they were immunostained with (A) anti-LC3B antibody (red) and (C) anti-p62 antibody (red). Nuclei were stained with DAPI. Images were taken under a confocal microscope. Scale bars: $10 \mu \mathrm{m}$. (B) and (D) Graphical representation of the percentage of cells showing the high intensity of LC3B and p62 respectively under Puma compromised conditions. Data represented were collected from of 4 independent experiments. Naïve PC12 cells were transfected with shFoxO3a-ZsGreen (green) or shRand-ZsGreen (green), primed, and then treated with $5 \mu \mathrm{M} A \beta$ for overnight after which they were immunostained with (E) anti-LC3B antibody (red) and (G) anti-p62 antibody (red). Nuclei were stained with DAPI. Images were taken under a confocal microscope. Scale bars: $10 \mu \mathrm{m}$. $(\mathbf{F})$ and $(\mathbf{H})$ Graphical representation of the percentage of cells showing the high intensity of LC3B and p62 respectively under FoxO3a compromised conditions. Data represented were collected from 4 independent experiments. Naïve PC12 cells were transfected with shPuma or shFoxO3a or shRand, primed, and then treated with $5 \mu \mathrm{M}$ A $\beta$ for overnight, and whole-cell lysates were assessed by western blot for protein levels of (I) LC3B and (M) p62 respectively. (J), (K) and (L) Graphical representation of the fold change of LC3B under A $\beta$ toxicity in control, Puma downregulation, and FoxO3a downregulation respectively. Data represented were collected from 4 independent experiments. (N), (O), (P) Graphical representation of the fold change of p62 under A $\beta$ toxicity in control, Puma downregulation, and FoxO3a downregulation respectively. Data represented were collected from 4 independent experiments. The asterisks denote statistically significant differences from control (shRand) at corresponding conditions; ${ }^{*} p<0.05,{ }^{* *} p<0.001,{ }^{* * *} p<0.0001$.

role of autophagy in neurodegeneration, we downregulated Beclin1 in neuronal cells and found that upon $A \beta$ treatment the viability of the neurons is better in Beclin1 downregulated conditions as compared to the $A \beta$ treated cells.

Interestingly, we found that knocking down of Puma in in vitro and in vivo significantly reduces the induction of autophagy but interestingly helps in maintaining the autophagic flux which is impaired in response to $A \beta$. This proves that proteins that are proapoptotic in nature or which help in directly activating the apoptotic process regulate autophagy in $A \beta$ induced toxicity by affecting its induction and flux. Alternatively, when autophagy inducer Beclin1 was downregulated, apoptosis is inhibited after $A \beta$ treatment. We could therefore infer that the inhibition of both the processes, viz, autophagy and apoptosis is beneficial for the neurons under $A \beta$ toxicity and that Puma has dual effects on autophagy. It reduces the induction of autophagy and secondly it promotes the flux of the already accumulated autophagic cargo, which eventually helps in the survival of the cells. We speculate that this regulation is brought about by a close interaction between Puma and Beclin1. The previous report has suggested an interaction between Bim and Beclin1 under starved conditions [41]. Our study reveals a direct interaction between Puma and Beclin1 upon $A \beta$ treatment. However, final outcome differs as Bim inhibits autophagy, whereas Puma induces autophagy but results in an impaired autophagic flux which causes neuron death. Further investigation is required to reveal this disparity between two similar molecules and to understand how Puma leads to impaired autophagic flux in AD.

\section{MATERIALS AND METHODS}

\section{Materials}

Lyophilized $A \beta_{1-42}$ was purchased from AlexoTech $A B$ (Umea, Sweden). 1,1,1,3,3,3 hexafluoro-2-propanol (HFIP), insulin, progesterone, transferrin, 
(A)

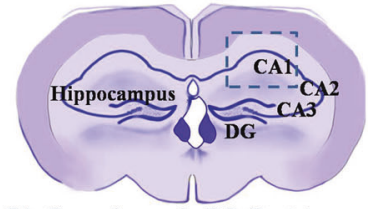

CA: Cornu Ammonis; DG: Dentate gyrus

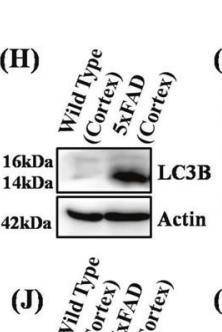

(I)

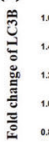

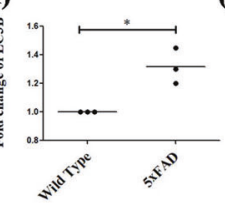

(K)

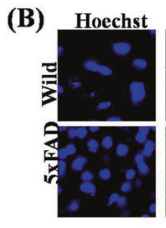

(D)

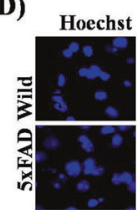

(F)

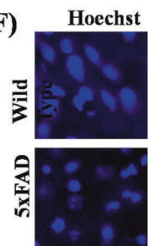

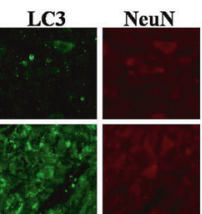

Lamp1
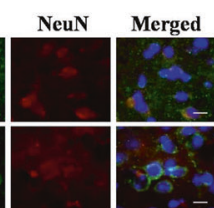

Puma

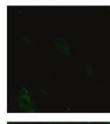

NeuN

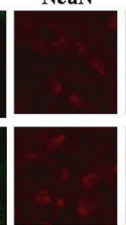

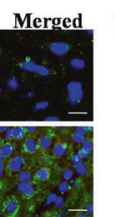

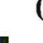

(1)

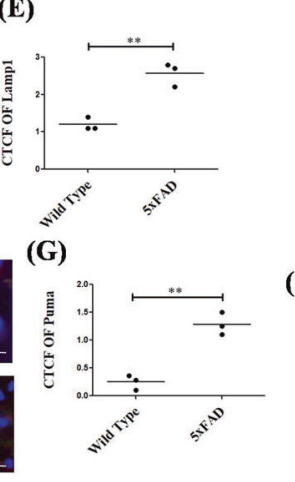

(C)

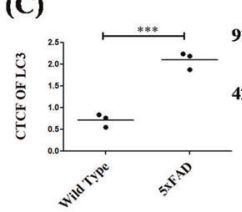

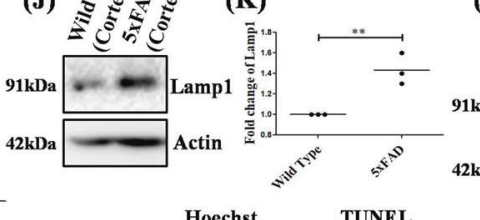

(L)

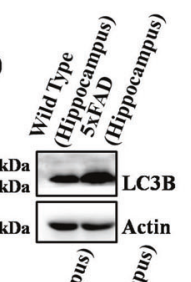

(M)

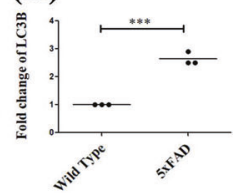

(N) 害离

(0)
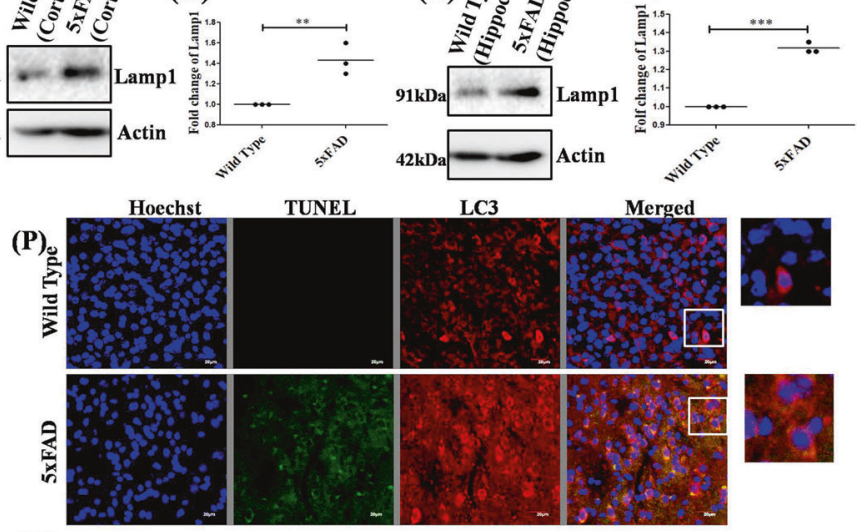

(Q)

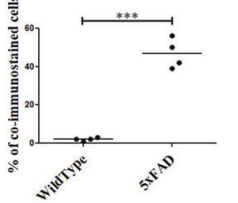

Fig. 6 Apoptosis and autophagy are induced in 5XFAD mice brains. (A) Schematic representation of the brain section depicting the Cornu Ammonis 1 (CA1) region from where the microscopic images were collected. Brain sections (20 micron) from the CA1 region of 6 months old 5XFAD mice and wild type mice were taken and stained with Hoechst (blue) for nuclei, neuronal marker NeuN (red) and (B) anti-LC3B antibody (green) (D) anti-Lamp1 antibody (green) (F) anti-Puma antibody (green) respectively. Scale bars: $38 \mu \mathrm{m}$. (C), (E), and (G) Graphical representation of the corrected total cell fluorescence of LC3B, Lamp1, and Puma, respectively. $n=3$ mice per group. Data represented were collected from 3 independent experiments. $(\mathbf{H})$ and $(\mathbf{J})$ represent western blot analysis of change in protein levels of LC3B and Lamp1 respectively of tissue lysates from the cortical region of 5xFAD mice brains as compared to the age-matched wild type mice. (I) and (K) Graphical representations of the fold change in autophagy proteins LC3B and Lamp1 in the cortical tissues of 5xFAD mice as compared to the wild-type mice. $n=3$ mice per group. Data represented were collected from 3 independent experiments. (L) and (N) represent western blot analysis of change in protein levels of LC3B and Lamp1, respectively of tissue lysates from the hippocampal region of 5xFAD mice brains as compared to the age-matched wild type mice. $(\mathbf{M})$ and $(\mathbf{O})$ Graphical representations of the fold change in autophagy proteins LC3B and Lamp1 in the hippocampal tissues of 5xFAD mice as compared to the wild-type mice. $n=3$ mice per group. Data represented were collected from 3 independent experiments. (P) Brain sections (20 micron) of 6 months5xFADand age-matched wild-type mice were immunostained with anti-LC3B antibody (red) and subjected to TUNEL assay (green). Scale bars: $20 \mu \mathrm{m}$. (Q) Graphical representation of the percentage costained cells in 5xFAD mice brains as compared to wild-type mice. $n=4$ mice per group. Data represented were collected from 4 independent experiments. The asterisks denote statistically significant differences from wild type to transgenic; ${ }^{*} p<0.05,{ }^{* *} p<0.001,{ }^{* * *} p<0.0001$.

human recombinant nerve growth factor (NGF), poly-D-lysine, putrescine, and selenium were purchased from Sigma (St. Louis, MO, USA). Anticleaved PARP (\#9542 T) and anti-pH2AX (\#2577) antibodies were from Cell Signaling Technology (Denver, MA, USA). Anti-p62 (\#MAB 8028) antibody was purchased from R\&D Systems (Minneapolis, MN, USA) and anti-Lamp1 was from Abcam (Cambridge, UK). Anti-LC3 (\#NB100-2220), anti-Beclin1 (\#NB500-249), anti-Puma (NBP1-76639) and anti- cleaved caspase 3 (\#NB100-56113) antibodies was purchased from Novus (Colorado, USA). Anti-actin $\beta$ (\#A3854) was procured for Sigma Aldrich (St. Louis, MO, USA). Anti-NeuN (MAB 377) was purchased from Millipore (Burlington, MA, USA). Anti-MAP2 antibody (sc-74421), Protein A agarose were from Santa Cruz Biotechnology (Dallas, Texas, USA). HRP conjugated secondary antibodies (\#7074 S and \#7076 S) were procured from Cell Signaling Technology (Denver, MA, USA). 4', 6-diamidino-2-phenylindole (DAPI), Lipofectamine 2000, Alexa Fluor 488 (\#A11008 and \#A11001), Alexa Fluor 546 (\#A11010 and \#A11003), culture media, and serum were purchased from Invitrogen, (Life technologies, Grand Island, NY, USA). Rapamycin (\#55310) and zVADFMK (\#627610) were procured from Calbiochem (CA, USA). Brain tissues of APPswe-PS1de9 (APP/PS1) mice and control littermates were kindly gifted by Dr. Anant B Patel [45].

\section{Cell culture}

Cortical neurons were isolated from the neocortex of E18 day embryonic rat brains and were cultured and maintained on poly-Dlysine-coated culture plates in DMEM/F12 medium with glucose $(6 \mathrm{mg} /$ $\mathrm{ml})$, progesterone $(20 \mathrm{ng} / \mathrm{ml})$, insulin $(25 \mu \mathrm{g} / \mathrm{ml})$, transferrin $(100 \mu \mathrm{g} / \mathrm{ml})$, putrescine $(60 \mu \mathrm{g} / \mathrm{ml})$ and selenium $(30 \mathrm{ng} / \mathrm{ml})$. The neurons were maintained for 6 days after which they were subjected to treatment $[46,47]$. Rat pheochromocytoma (PC12) cells (Research Resource Identifier, RRID: CVCL_0481) were cultured and maintained in DMEM medium (with glucose) supplemented with $10 \%$ heat-inactivated horse serum (HS) and 5\% heat-inactivated fetal bovine serum (FBS) [48]. Differentiation of PC12 cells were brought about by NGF $(50 \mathrm{ng} / \mathrm{ml})$ in a medium containing $1 \%$ horse serum for 5 days prior to treatment [49]. PC12 cells were authenticated using a neuron-specific marker (immunostained with anti-MAP2 antibody), before use and were maintained up to 30 passages. For primary and secondary cell lines, 3-4 independent cell samples were processed and all of them were used for experimental studies and analysis. For all experiments, the outcomes were crosschecked by a third person who was completely blinded towards the experimental conditions. 
(A)
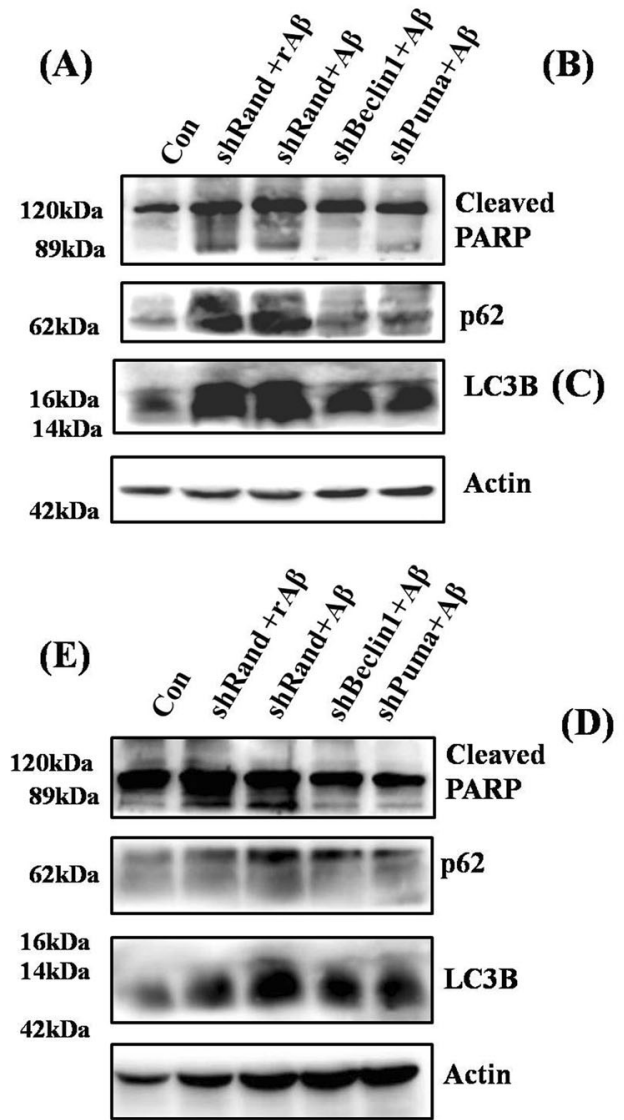
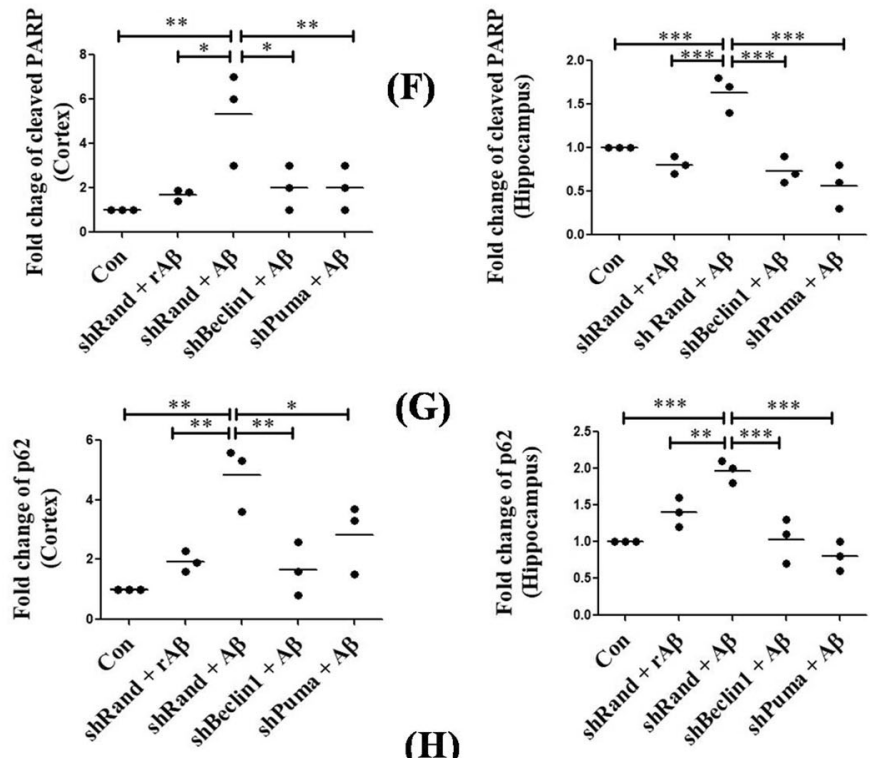

(G)

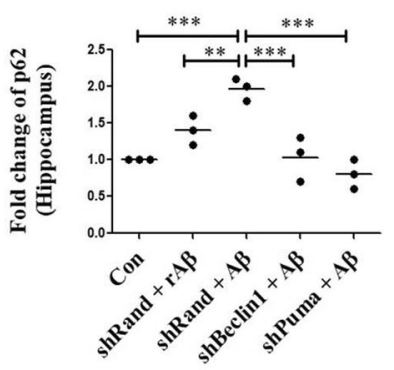

(H)

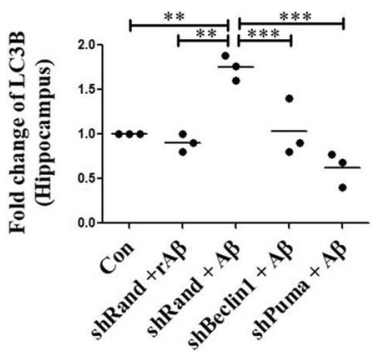

Fig. 7 Down regulation of autophagy BH3-only protein Beclin1 and pro-apoptotic BH3-only protein Puma helps in cell survival and in autophagy flux maintenance. (A) Brain tissue lysates from the cortical area infused with $A \beta$ along with shPuma or shBeclin1 were taken and assessed by western blot for LC3B, p62, and cleaved PARP. (B), (C) and (D) Graphical representations of the fold change of cleaved PARP, p62, and LC3B respectively in the cortical lysates of the animals. $n=3$ rats per group. Data represented were collected from 3 independent experiments. (E) Brain tissue lysates from the hippocampal region were taken and assessed by western blot for LC3B, p62 cleaved PARP. (F), (G) and (H) Graphical representations of the fold change of cleaved PARP, p62, and LC3B respectively in the hippocampal lysates of the animals. $n=3$ rats per group. Data represented were collected from 3 independent experiments. The asterisks denote statistically significant differences between corresponding conditions; ${ }^{*} p<0.05,{ }^{* *} p<0.001,{ }^{* * *} p<0.0001$.

\section{Animal models}

Adult male Sprague Dawley (SD) rats weighing around 300-380 g were used. The animals were housed a maximum of three per cage in a temperature-controlled room $\left(24 \pm 2^{\circ} \mathrm{C}\right)$, with $12-12 \mathrm{~h}$ light-dark cycle, humidity $(60 \pm 5 \%)$ and allowed to food and water ad libitum in the animal house of CSIR-Indian Institute of Chemical Biology, Kolkata. Adult 5xFAD mice (Swedish, Florida, and London mutations in APP and the M146L and L286V mutations in PSEN1 and age-matched wild type control C57BL/6 mice were procured from Jackson's Laboratory). The mice were housed a maximum of five mice per cage in a temperaturecontrolled room and 12-12 h light-dark cycle, humidity as mentioned above. A set of 3 animals per group were used in the experiments since we performed only biochemical analysis.

\section{Preparation of $A \boldsymbol{\beta}$ oligomers}

Purified and lyophilized $A \beta_{1-42}$ powder was reconstituted in HFIP to a concentration of $1 \mathrm{mM}$. The reconstituted solution was subjected to Speed Vac for 30-35 min for the complete removal of HFIP by evaporation. The obtained pellet was then resuspended in anhydrous DMSO to a concentration of $5 \mathrm{mM}$ followed by sonication in a water bath for $10 \mathrm{~min}$ at $37^{\circ} \mathrm{C}$. This stock solution was then stored at $-80^{\circ} \mathrm{C}$. The stock solution was further processed and diluted to an intermittent concentration of $400 \mu \mathrm{M}$ using PBS and SDS was added to a final concentration of $0.2 \%$. This solution was incubated at $37^{\circ} \mathrm{C}$ for $18-24 \mathrm{~h}$. The preparation was further diluted to a final concentration of $100 \mu \mathrm{M}$ using PBS and incubated at $37^{\circ} \mathrm{C}$ for $18-24 \mathrm{~h}$ before use.

\section{Oligomeric $A \boldsymbol{\beta}$ treatment to cells}

Freshly prepared oligomeric $A \beta_{1-42}$ was added to the medium containing neuronal cells for the specific time points as required. For neuronally differentiated PC12 cells, the concentration of oligomeric $A \beta_{1-42}$ used was $5 \mu \mathrm{M}$ and for primary rat cortical neurons, the concentration used was $1.5 \mu \mathrm{M}[33,34]$.

\section{Western Blot analysis}

Treated and untreated cortical neurons or neuronally differentiated PC12 cells were lysed and proteins were analyzed by western blotting [50]. 30-50 $\mu \mathrm{g}$ of protein for each condition was resolved in 8-12\% SDS-PAGE as per the need followed by their transfer on to PVDF membrane (Hybond: GE Healthcare, Buckinghamshire, UK). The proteins were then probed with the desired primary antibodies at $4{ }^{\circ} \mathrm{C}$ for overnight. HRP-conjugated secondary antibodies against the primary antibodies were used. The blots were detected using Biorad western blotting detection reagent, according to the manufacturer's protocol. Imaging of all Western blots was performed using an Azure Chemidoc system using manufacturer's protocol.

\section{Immunocytochemical staining}

Neuronally differentiated PC12 cells and/or primary cortical neurons were fixed with $4 \%$ paraformaldehyde for $10 \mathrm{~min}$. The cells were washed thrice with PBS for 5 min each, thoroughly. The cells were then blocked in $3 \%$ goat serum in PBS containing $0.3 \%$ Triton-X 100 at room temperature for $2 \mathrm{~h}$. The cells were then immunolabelled with the desired primary antibody in the blocking solution for overnight at $4^{\circ} \mathrm{C}$. 


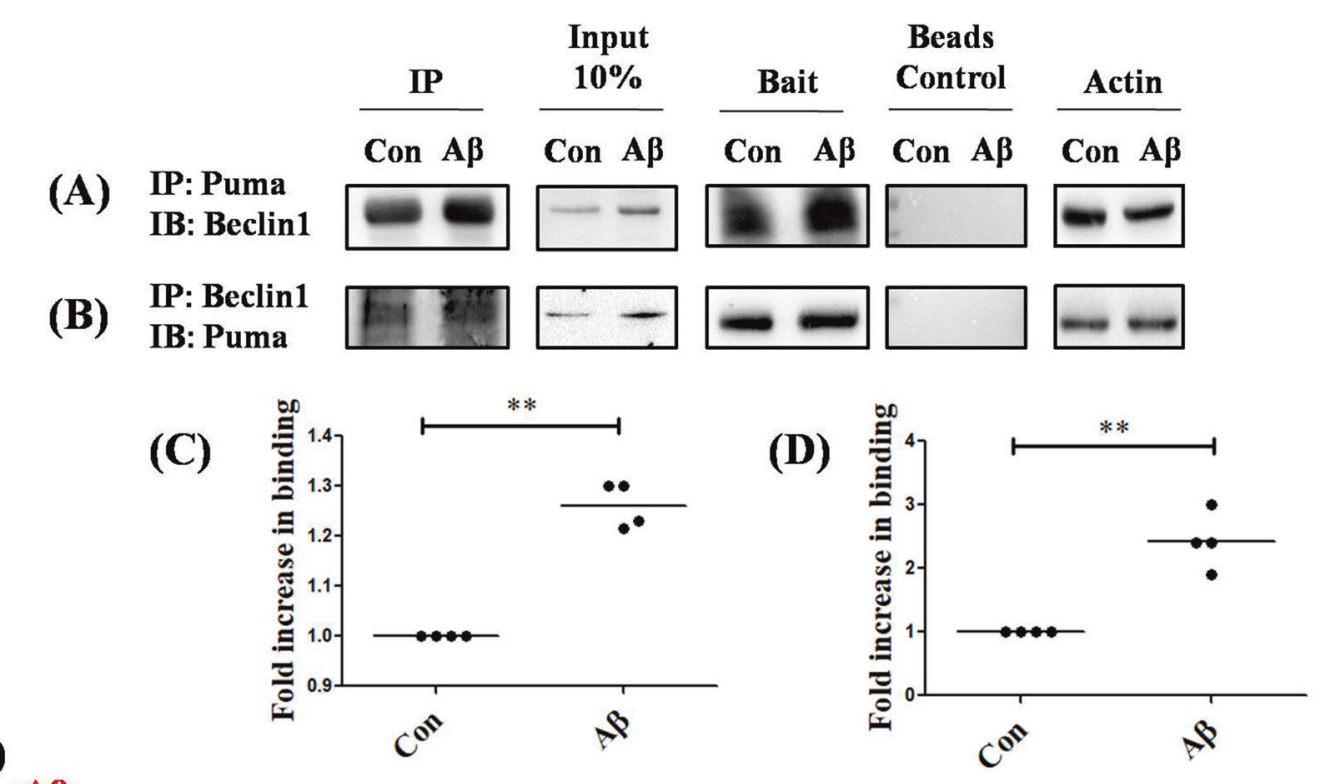

(E)

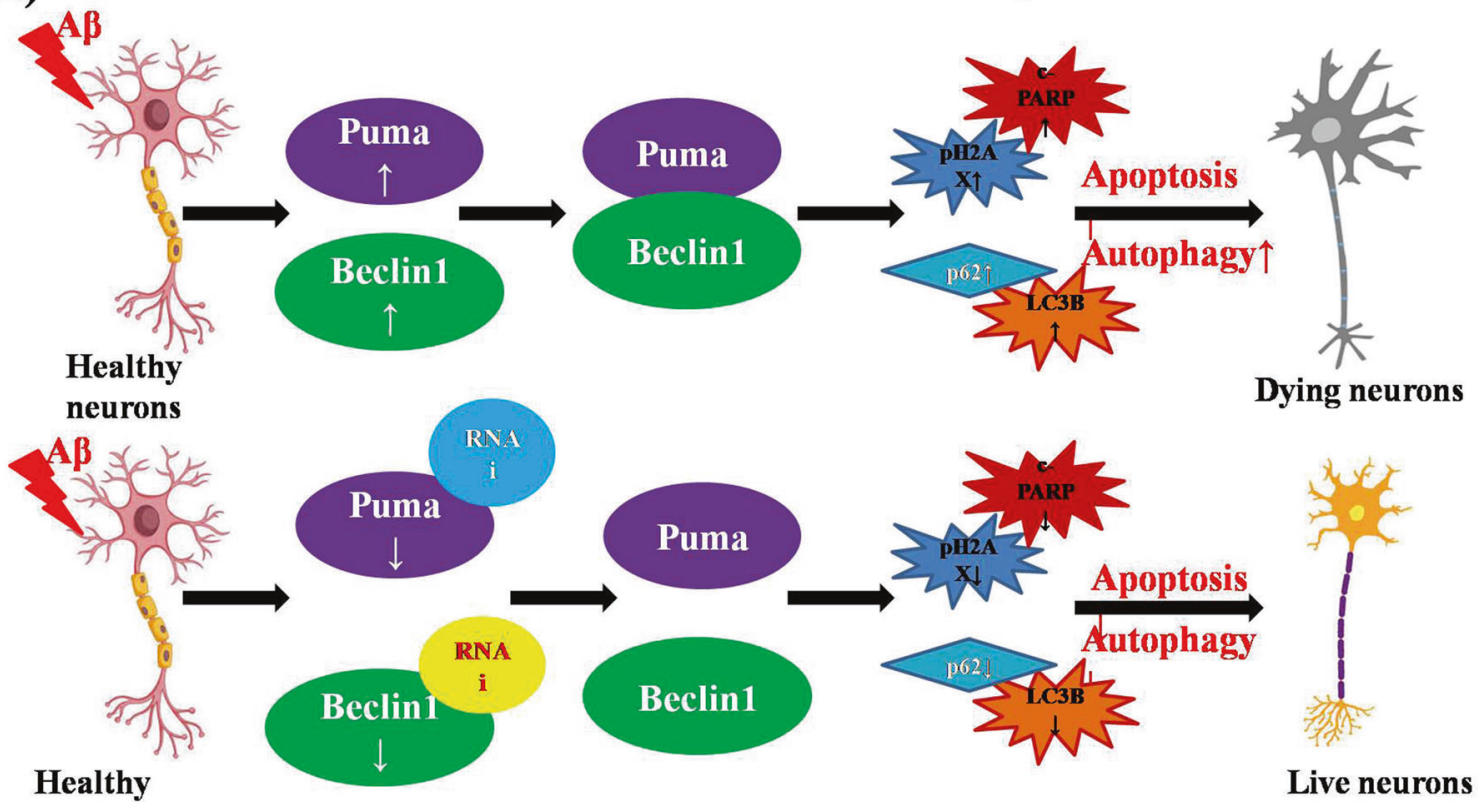

neurons

Fig. 8 Autophagic BH3-only protein Beclin1 interacts with pro-apoptotic BH3-only protein Puma in cortical neurons. A Primary rat cortical neurons were cultured and treated with and without $1.5 \mu \mathrm{M} \mathrm{A} \beta$ for overnight and the cell lysates were immunoprecipitated (IP) with Puma antibody and immunoblotted (IB) for Beclin1. B Reverse immunoprecipitation was also performed to check the interaction of Beclin1 with Puma; cell lysates were immunoprecipitated with Beclin1 antibody and immunoblotted for Puma. The inputs and baits for the respective immunoprecipitation studies are shown in the second and the third column of $(\mathbf{A})$ and $(\mathbf{B})$. The beads controls are shown in the fourth column and the loading controls (Actin) for each immunoprecipitation study are represented in the fifth column of (A) and (B). (C) and (D) Graphical representation of the fold change in the interaction between Beclin 1 and Puma under A $\beta$ treatment as compared to control. Data represented were collected from 4 independent experiments. The asterisks denote statistically significant differences between corresponding conditions; ${ }^{* *} p<0.001$. E Schematic representation depicting the role of BH3-only proteins Puma and Beclin 1 in the regulation of autophagic death in neurons exposed to $A \beta$.

The next day, the cells were washed in PBS thrice for 5-10 min each, followed by incubation with the appropriate secondary antibody for $2 \mathrm{~h}$ at room temperature. Nuclei were stained with Hoechst or DAPI. Highresolution images were taken using Leica TCS SP8 microscope (Wetzlar, Germany). For co-immunocytochemistry, the cells were first incubated with TUNEL reagent as per manufacturer's protocol followed by incubation with primary and secondary antibodies. Fluorescence intensities of staining for control or treated cells were quantified using $\mathrm{NIH}$ ImageJ software. The corrected total cell fluorescence (CTCF) was determined by considering the integrated density of staining, area of the cell, and the background fluorescence for the different experimental conditions. CTCF $=$ Integrated density - (area of selected cell $\times$ mean fluorescence of background readings).

\section{Immunoprecipitation}

Primary cultured cortical neurons were either treated with $1.5 \mu \mathrm{M} \mathrm{A} \beta$ for $16 \mathrm{~h}$ (overnight) or left as untreated controls and interactions of Beclin1 with Puma were detected by co-immunoprecipitation assays. The cells were washed with PBS thrice for 5 min each and lysed. For immunoprecipitation, 
the agarose-conjugated anti-Beclin 1 antibody was prepared by incubating $3 \mu \mathrm{g}$ of anti-Beclin1 antibody with $20 \mu \mathrm{l}$ of protein A agarose beads at $4{ }^{\circ} \mathrm{C}$ for $2 \mathrm{~h}$ in a shaking condition. The agarose-conjugated Beclin 1 antibody was then pooled down and incubated with treated and untreated cell lysates containing equal amounts of protein for overnight at $4{ }^{\circ} \mathrm{C}$ on a shaker. Following this incubation, antigen-antibody complexes were isolated and dissociated by boiling in sample buffer for $4 \mathrm{~min}$. The agarose beads were centrifuged and the supernatant was subjected to western blot analysis as described previously, for the expression of Puma.

\section{Transfection}

DNA was isolated using a Plasmid Maxi kit (Qiagen, Waltham, MA, USA). For survival assay, PC12 cells and/or primary cortical neurons were transfected with $0.5 \mu \mathrm{g}$ of either pSIREN-Puma-shRNA-ZsGreen (shPuma) or pSIRENFoxO3a-shRNA-ZsGreen (shFoxO3a)or pSIREN-Beclin1-shRNA-ZsGreen (shBeclin1) or pSIREN-Rand-shRNA-ZsGreen (shRand). Transfections were performed using lipofectamine 2000 in $500 \mu \mathrm{l}$ of a serum-free medium in each well of a 24-well plate. Lipofectamine-containing medium was replaced by fresh medium after $4-6 \mathrm{~h}$ of transfection. Transfection was performed on the third day of culture for primary cortical neurons. Differentiated neuronal PC12 cells were transfected on the third day of differentiation. For endogenous Puma or Beclin1or FoxO3adownregulation, naive PC12 cells were transfected with either $1.5 \mu \mathrm{g}$ of shPuma or shBeclin1 or shFoxO3a or shRand, followed by differentiation and treatment. Transfections were done in $1.5 \mathrm{ml}$ of serum-free medium per well of a 6-well plate using lipofectamine 2000.

\section{Survival Assay}

Primary cortical neurons (3DIV) and differentiated PC12 cells (3DIV) were transfected with shRand or shBeclin 1 as mentioned above. The cells were exposed to oligomeric $A \beta_{1-42}$ after $48 \mathrm{~h}$ of transfection. The number of transfected neurons (green) was counted $(0 \mathrm{~h})$ under the microscope. The transfected and surviving neurons were again counted after $24 \mathrm{~h}$ and $48 \mathrm{~h}$ of treatment [49]. Control and $A \beta$-treated transfected neurons were imaged under Leica TCS SP8 microscope (Wetzlar, Germany).

\section{TUNEL assay}

TUNEL positive cells in $A \beta_{1-42}$ treated PC12 cells or in brain sections of transgenic mice models were detected using the in-situ cell death detection kit (Clonetech, CA, USA). The steps involved in the process were followed as given in the manufacturer's protocol.

\section{Oligomeric $A \beta$ infusion in animals}

Male Sprague Dawley (SD) rats (300-380 g) were infused with oligomeric $A \beta$ as described previously [51]. Rats were anaesthetized by injecting a mixture of xylazine-ketamine, placed on a stereotaxic frame. A volume of $5 \mu \mathrm{l}$ of $100 \mu \mathrm{M}$ A $\beta$ in PBS was infused in the right cerebral cortex at stereotaxic co-ordinates from bregma: AP: $-4.1, \mathrm{ML}: 2.5, \mathrm{DV}: 1.3 \mathrm{~mm}$, according to the rat brain atlas and a previous report [51]. An equal volume of PBS was injected in control animals. $2 \mu \mathrm{g}$ of pSIREN-Puma-shRNAZsGreen (shPuma) or pSIREN-Beclin1-shRNA-ZsGreen (shBeclin1) or pSIREN-Rand-shRNA-ZsGreen (shRand) was infused in the rat cortices along with the $A \beta$ to create Puma-deficient, Beclin1-deficient and sham control animal models of $A D$ [52]. Reverse amyloid-beta peptide, $A \beta_{42-1}$ $(\mathrm{rA} \beta)$ was also injected in similar loci of the rat brains to create a negative control model. Randomizations of the animals were done for the above groups such that similar age and weighted animals were selected for the stereotactic infusion. After infusion, every animal was considered for the experimentation. No animals were excluded for the present study. Animals have sacrificed 11 days post-injection. The brains were dissected out following cardiac perfusion and fixed in 4\% PFA for $24 \mathrm{~h}$ and were then incubated in a $30 \%$ sucrose solution for $24 \mathrm{~h}$ and then cryo-sectioning was performed by using a cryotome (Thermo, West Palm Beach, FL, USA). Collection of tissue samples for western blot analysis and sectioning were done blindly. Tissue samples for western blot analysis were taken from the cortical and hippocampal regions of dissected and non-perfused brains and subjected to tissue homogenization and quantification.

\section{Immunohistochemistry of brain slices}

Cryo sections measuring $20 \mu \mathrm{m}$ of the brains from A $\mathrm{A}$-infused or PBSinfused rats (as described earlier) or wild-type or APP/PS1 (Swedish mutation in APP and PS1 mutation) or 5xFAD transgenic mice were taken and blocked with $5 \%$ goat serum in PBS containing $0.3 \%$ Triton-X 100 for $1 \mathrm{~h}$ at room temperature. The sections were then incubated with the desired primary antibody in a blocking solution for overnight at $4{ }^{\circ} \mathrm{C}$. The following day, the sections were washed with PBS thrice and then incubated with a fluorescence-tagged secondary antibody for $2 \mathrm{~h}$ at room temperature. The nuclei were stained with Hoechst or DAPI. The sections were mounted in anti-fade reagent and high-resolution images were taken using Leica TCS SP8 microscope. For co-immunohistochemistry, the sections were first incubated with TUNEL reagent as per manufacturer's protocol followed by incubation with primary and secondary antibodies.

\section{Statistics}

Experimental results are reported as the mean of three individual experiments using GraphPad Prism 5 software. Student's t-test was performed as paired or unpaired wherever required, two-tailed sets of arrays to evaluate the significance of the difference between the means and is presented as p-values. For multiple conditions, one-way ANOVA was performed wherever required, to evaluate the significance of the difference between the means and are presented as p-values. NewmanKeuls Multiple Comparison post-test was performed. 95\% confidence interval was considered for the significance. For survival assays, two-way ANOVA was performed and Bonferroni post-tests were performed to evaluate the variance and significance which is presented as $p$-values.

\section{DATA AVAILABILITY}

The datasets used and/or analyzed during the current study are available from the corresponding author on reasonable request.

\section{REFERENCES}

1. Hardy JA, Higgins GA. Alzheimer's disease: the amyloid cascade hypothesis. Science. 1992;256:184-5.

2. Hardy J, Selkoe DJ. The amyloid hypothesis of Alzheimer's disease: progress and problems on the road to therapeutics. Science 2002;297:353-6.

3. De Strooper B, Karran E. The cellular phase of Alzheimer's disease. Cell. 2016;164:603-15.

4. Long JM, Holtzman DM. Alzheimer disease: an update on pathobiology and treatment strategies. Cell 2019;179:312-39.

5. Yuan J, Lipinski M, Degterev A. Diversity in the mechanisms of neuronal cell death. Neuron 2003;40:401-13.

6. Fricker M, Tolkovsky AM, Borutaite V, Coleman M, Brown GC. Neuronal Cell Death Physiol. Rev. 2018;98:813-80.

7. Saleem S. Apoptosis, Autophagy, Necrosis and Their Multi Galore Crosstalk in Neurodegeneration. Neuroscience 2021;469:162-74.

8. Levine B, Kroemer G. Autophagy in the pathogenesis of disease. Cell 2008:132:27-42.

9. Dunn WA Jr. Autophagy and related mechanisms of lysosome-mediated protein degradation. Trends Cell Biol. 1994;4:139-43.

10. Mortimore GE, Lardeux BR, Heydrick SJ. Mechanism and control of protein and RNA degradation in the rat hepatocyte: two modes of autophagic sequestration. Revis. Biol. Cel. 1989;20:79-96.

11. Ghavami S, Shojaei S, Yeganeh B, Ande SR, Jangamreddy JR, Mehrpour M, et al. Autophagy and apoptosis dysfunction in neurodegenerative disorders. Prog Neurobiol. 2014;112:24-49.

12. Tooze SA, Schiavo G. Liaisons dangereuses: autophagy, neuronal survival and neurodegeneration. Curr. Opin. Neurobiol. 2008;18:504-15.

13. Yap CC, Winckler B. Harnessing the power of the endosome to regulate neural development. Neuron 2012;74:440-51.

14. Nixon RA. The role of autophagy in neurodegenerative disease. Nat. Med. 2013;19:983-97.

15. Hara T, Nakamura $K$, Matsui $M$, Yamamoto A, Nakahara $Y$, Suzuki-Migishima $R$, et al. Suppression of basal autophagy in neural cells causes neurodegenerative disease in mice. Nature 2006;441:885-9.

16. Komatsu M, Waguri S, Chiba T, Murata S, Iwata J, Tanida I, et al. Loss of autophagy in the central nervous system causes neurodegeneration in mice. Nature 2006:441:880-4.

17. Kuma A, Komatsu M, Mizushima N. Autophagy-monitoring and autophagydeficient mice. Autophagy 2017;13:1619-28.

18. Wertz MH, Mitchem MR, Pineda SS, Hachigian LJ, Lee H, Lau V, et al. Genomewide In Vivo CNS Screening Identifies Genes that Modify CNS Neuronal Survival and mHTT Toxicity. Neuron 2020;106:76-89.

19. Liang JH, Jia JP. Dysfunctional autophagy in Alzheimer's disease: pathogenic roles and therapeutic implications. Neurosci. Bull. 2014;30:308-16. 
20. Nilsson $P$, Loganathan $K$, Sekiguchi $M$, Matsuba $Y$, Hui $K$, Tsubuki $S$, et al. Abeta secretion and plaque formation depend on autophagy. Cell Rep. 2013;5:61-9.

21. Tian Y, Bustos V, Flajolet M, Greengard P. A small-molecule enhancer of autophagy decreases levels of Abeta and APP-CTF via Atg5-dependent autophagy pathway. FASEB J. 2011;25:1934-42.

22. Cho MH, Cho K, Kang HJ, Jeon EY, Kim HS, Kwon HJ, et al. Autophagy in microglia degrades extracellular beta-amyloid fibrils and regulates the NLRP3 inflammasome. Autophagy 2014;10:1761-75.

23. Ries M, Sastre M. Mechanisms of Abeta clearance and degradation by glial cells. Front Aging Neurosci. 2016;8:160.

24. Wei Y, Zhou J, Wu J, Huang J. ERbeta promotes Abeta degradation via the modulation of autophagy. Cell Death Dis. 2019;10:565.

25. Lee MJ, Lee $\mathrm{JH}$, Rubinsztein DC. Tau degradation: the ubiquitin-proteasome system versus the autophagy-lysosome system. Prog. Neurobiol. 2013;105:49-59.

26. Jiang S, Bhaskar K. Degradation and transmission of tau by autophagicendolysosomal networks and potential therapeutic targets for tauopathy. Front Mol. Neurosci. 2020;13:586731.

27. Silva MC, Nandi GA, Tentarelli S, Gurrell IK, Jamier T, Lucente D, et al. Prolonged tau clearance and stress vulnerability rescue by pharmacological activation of autophagy in tauopathy neurons. Nat. Commun. 2020;11:3258.

28. Ling D, Salvaterra PM. A central role for autophagy in Alzheimer-type neurodegeneration. Autophagy 2009;5:738-40.

29. Funderburk SF, Wang QJ, Yue Z. The Beclin 1-VPS34 complex-at the crossroads of autophagy and beyond. Trends Cell Biol. 2010;20:355-62.

30. Uddin MS, Stachowiak A, Mamun AA, Tzvetkov NT, Takeda S, Atanasov AG, et al. Autophagy and Alzheimer's disease: from molecular mechanisms to therapeutic implications. Front Aging Neurosci. 2018;10:04.

31. Liu J, Li L. Targeting autophagy for the treatment of alzheimer's disease: challenges and opportunities. Front Mol. Neurosci. 2019;12:203.

32. Morishima Y, Gotoh Y, Zieg J, Barrett T, Takano H, Flavell R, et al. Beta-amyloid induces neuronal apoptosis via a mechanism that involves the $\mathrm{c}$-Jun $\mathrm{N}$-terminal kinase pathway and the induction of Fas ligand. J. Neurosci. 2001;21:7551-60.

33. Sanphui $\mathrm{P}$, Biswas SC. FoxO3a is activated and executes neuron death via Bim in response to beta-amyloid. Cell Death Dis. 2013;4:e625.

34. Akhter R, Sanphui P, Biswas SC. The essential role of p53-up-regulated modulator of apoptosis (Puma) and its regulation by FoxO3a transcription factor in betaamyloid-induced neuron death. J. Biol. Chem. 2014;289:10812-22.

35. Akhter R, Sanphui P, Das H, Saha P, Biswas SC. The regulation of p53 up-regulated modulator of apoptosis by JNK/C-Jun pathway in beta-amyloid-induced neuron death. J. Neurochem. 2015;134:1091-103.

36. Akhter R, Saleem S, Saha A, Biswas SC. The pro-apoptotic protein Bmf co-operates with Bim and Puma in neuron death induced by beta-amyloid or NGF deprivation. Mol. Cell Neurosci. 2018;88:249-57.

37. Shimohama S. Apoptosis in Alzheimer's disease-an update. Apoptosis 2000;5:9-16.

38. Behl C. Apoptosis and Alzheimer's disease. J. Neural Transm. 2000;107:1325-44.

39. Maiuri MC, Zalckvar E, Kimchi A, Kroemer G. Self-eating and self-killing: crosstalk between autophagy and apoptosis. Nat. Rev. Mol. Cell Biol. 2007;8:741-52.

40. Thorburn A. Apoptosis and autophagy: regulatory connections between two supposedly different processes. Apoptosis 2008;13:1-9.

41. Luo S, Garcia-Arencibia M, Zhao R, Puri C, Toh PP, Sadiq O, et al. Bim inhibits autophagy by recruiting Beclin 1 to microtubules. Mol. Cell. 2012;47:359-70.

42. Pattingre S, Tassa A, Qu X, Garuti R, Liang XH, Mizushima N, et al. BCl-2 antiapoptotic proteins inhibit Beclin 1-dependent autophagy. Cell 2005;122:927-39.

43. Wirawan E, Vande Walle L, Kersse K, Cornelis S, Claerhout S, Vanoverberghe I, et al. Caspase-mediated cleavage of Beclin-1 inactivates Beclin-1-induced autophagy and enhances apoptosis by promoting the release of proapoptotic factors from mitochondria. Cell Death Dis.2010;1:e18.

44. Djavaheri-Mergny M, Maiuri MC, Kroemer G. Cross talk between apoptosis and autophagy by caspase-mediated cleavage of Beclin 1. Oncogene 2010;29:1717-9. Mar 25

45. Tiwari $V$, Patel $A B$. Impaired glutamatergic and GABAergic function at early age in AbetaPPswe-PS1dE9 mice: implications for Alzheimer's disease. J. Alzheimers Dis. 2012;28:765-9.

46. Park DS, Morris EJ, Padmanabhan J, Shelanski ML, Geller HM, Greene LA. Cyclindependent kinases participate in death of neurons evoked by DNA-damaging agents. J. Cell Biol. 1998;143:457-67.

47. Troy CM, Rabacchi SA, Friedman WJ, Frappier TF, Brown K, Shelanski ML. Caspase2 mediates neuronal cell death induced by beta-amyloid. J. Neurosci. 2000;20:1386-92.

48. Greene LA, Tischler AS. Establishment of a noradrenergic clonal line of rat adrenal pheochromocytoma cells which respond to nerve growth factor. Proc. Natl Acad. Sci. USA. 1976;73:2424-8

49. Biswas SC, Shi Y, Vonsattel JP, Leung CL, Troy CM, Greene LA. Bim is elevated in Alzheimer's disease neurons and is required for beta-amyloid-induced neuronal apoptosis. J. Neurosci. 2007;27:893-900.
50. Biswas SC, Greene LA. Nerve growth factor (NGF) down-regulates the Bcl-2 homology $3(\mathrm{BH} 3)$ domain-only protein Bim and suppresses its proapoptotic activity by phosphorylation. J. Biol. Chem. 2002;277:49511-6.

51. Frautschy SA, Baird A, Cole GM. Effects of injected Alzheimer beta-amyloid cores in rat brain. Proc. Natl Acad. Sci. USA. 1991;88:8362-6.

52. De D, Mukherjee I, Guha S, Paidi RK, Chakrabarti S, Biswas SC, et al. Rheb-mTOR activation rescues Abeta-induced cognitive impairment and memory function by restoring miR-146 activity in glial cells. Mol. Ther. Nucleic Acids. 2021;24:868-87.

53. Erlich S, Shohami E, Pinkas-Kramarski R. Neurodegeneration induces upregulation of Beclin 1. Autophagy 2006;2:49-51.

54. Cao Y, Klionsky DJ. Physiological functions of Atg6/Beclin 1: a unique autophagyrelated protein. Cell Res. 2007;17:839-49.

55. Kang R, Zeh HJ, Lotze MT, Tang D. The Beclin 1 network regulates autophagy and apoptosis. Cell Death Differ. 2011;18:571-80.

56. Bieri G, Lucin KM, O'Brien CE, Zhang H, Villeda SA, Wyss-Coray T. Proteolytic cleavage of Beclin 1 exacerbates neurodegeneration. Mol. Neurodegener. 2018;13:68.

57. Panda PK, Naik PP, Meher BR, Das DN, Mukhopadhyay S, Praharaj PP, et al. PUMA dependent mitophagy by Abrus agglutinin contributes to apoptosis through ceramide generation. Biochim Biophys. Acta Mol. Cell Res. 2018;1865:480-95.

58. Sengupta A, Molkentin JD, Yutzey KE. FoxO transcription factors promote autophagy in cardiomyocytes. J. Biol. Chem. 2009;284:28319-31.

59. Oberstein A, Jeffrey PD, Shi Y. Crystal structure of the Bcl-XL-Beclin 1 peptide complex: Beclin 1 is a novel BH3-only protein. J. Biol. Chem. 2007;282:13123-32.

60. Pyo JO, Jang MH, Kwon YK, Lee HJ, Jun Jl, Woo HN, et al. Essential roles of Atg5 and FADD in autophagic cell death: dissection of autophagic cell death into vacuole formation and cell death. J. Biol. Chem. 2005;280:20722-9.

61. Yousefi S, Perozzo R, Schmid I, Ziemiecki A, Schaffner T, Scapozza L, et al. Calpainmediated cleavage of Atg5 switches autophagy to apoptosis. Nat. Cell Biol. 2006;8:1124-32.

62. Thorburn J, Andrysik Z, Staskiewicz L, Gump J, Maycotte P, Oberst A, et al. Autophagy controls the kinetics and extent of mitochondrial apoptosis by regulating PUMA levels. Cell Rep. 2014;7:45-52.

63. Anderson FL, von Herrmann KM, Young AL, Havrda MC. Bbc3 loss enhances survival and protein clearance in neurons exposed to the organophosphate pesticide chlorpyrifos. Toxicol Sci. 2021.

64. Ginet V, Puyal J, Clarke PG, Truttmann AC. Enhancement of autophagic flux after neonatal cerebral hypoxia-ischemia and its region-specific relationship to apoptotic mechanisms. Am. J. Pathol. 2009;175:1962-74. Nov

65. Nixon RA, Wegiel J, Kumar A, Yu WH, Peterhoff C, Cataldo A, et al. Extensive involvement of autophagy in Alzheimer disease: an immuno-electron microscopy study. J. Neuropathol. Exp. Neurol. 2005;64:113-22.

66. Yu WH, Kumar A, Peterhoff C, Shapiro Kulnane L, Uchiyama Y, Lamb BT, et al. Autophagic vacuoles are enriched in amyloid precursor protein-secretase activities: implications for beta-amyloid peptide over-production and localization in Alzheimer's disease. Int J. Biochem Cell Biol. 2004;36:2531-40.

67. Yang DS, Kumar A, Stavrides P, Peterson J, Peterhoff CM, Pawlik M, et al. Neuronal apoptosis and autophagy cross talk in aging PS/APP mice, a model of Alzheimer's disease. Am. J. Pathol. 2008;173:665-81.

68. Saleem S, Biswas SC. Tribbles pseudokinase 3 induces both apoptosis and autophagy in amyloid-beta-induced neuronal death. J. Biol. Chem. 2017:292:2571-85.

\section{ACKNOWLEDGEMENTS}

The work was supported by the Department of Science and Technology, Govt. of India, Project EMR/2016/003312, and CSIR, Govt. of India. We thank Ms. Naqiya Ambareen for her critical reading of the manuscript.

\section{AUTHOR CONTRIBUTIONS}

A.S., S.S., and S.C.B. conceived and designed the study and S.C.B. supervised the project. A.S. performed biochemical, molecular, and histological experiments. A.S and S.S. performed cell culture studies. A.S. and R.K.P. performed the animal stereotaxy and tissue sectioning. A.S., S.S, and S.C.B analyzed the data and contributed to writing the paper.

\section{COMPETING INTERESTS}

The authors declare no competing interests.

\section{ETHICS STATEMENT}

All animal studies were carried out in accordance with the guidelines formulated by the Committee for the Purpose of Control and Supervision of Experiments on Animals (Animal Welfare Divisions, Ministry of Environments and Forests, Govt. of 
India) with approval from the Institutional Animal Ethics Committee (IAEC). We have consulted the ARRIVE guidelines for the relevant aspects of animal studies.

\section{ADDITIONAL INFORMATION}

Supplementary information The online version contains supplementary material available at https://doi.org/10.1038/s41420-021-00748-x.

Correspondence and requests for materials should be addressed to Subhas C. Biswas.

Reprints and permission information is available at http://www.nature.com/ reprints

Publisher's note Springer Nature remains neutral with regard to jurisdictional claims in published maps and institutional affiliations.
Open Access This article is licensed under a Creative Commons Attribution 4.0 International License, which permits use, sharing, adaptation, distribution and reproduction in any medium or format, as long as you give appropriate credit to the original author(s) and the source, provide a link to the Creative Commons license, and indicate if changes were made. The images or other third party material in this article are included in the article's Creative Commons license, unless indicated otherwise in a credit line to the material. If material is not included in the article's Creative Commons license and your intended use is not permitted by statutory regulation or exceeds the permitted use, you will need to obtain permission directly from the copyright holder. To view a copy of this license, visit http://creativecommons. org/licenses/by/4.0/.

(c) The Author(s) 2021, corrected publication 2021 